\title{
Nondifferentiable Minimax Programming Problems in Complex Spaces Involving Generalized Convex Functions
}

\author{
Anurag Jayswal, Ashish Kumar Prasad, and Krishna Kummari \\ Department of Applied Mathematics, Indian School of Mines, Dhanbad, Jharkhand 826 004, India \\ Correspondence should be addressed to Anurag Jayswal; anurag_jais123@yahoo.com
}

Received 17 June 2013; Accepted 7 November 2013

Academic Editor: Sheng-Jie Li

Copyright ( 2013 Anurag Jayswal et al. This is an open access article distributed under the Creative Commons Attribution License, which permits unrestricted use, distribution, and reproduction in any medium, provided the original work is properly cited.

\begin{abstract}
We start our discussion with a class of nondifferentiable minimax programming problems in complex space and establish sufficient optimality conditions under generalized convexity assumptions. Furthermore, we derive weak, strong, and strict converse duality theorems for the two types of dual models in order to prove that the primal and dual problems will have no duality gap under the framework of generalized convexity for complex functions.
\end{abstract}

\section{Introduction}

The literature of the mathematical programming is crowded with necessary and sufficient conditions for a point to be an optimal solution to the optimization problem. Levinson [1] was the first to study mathematical programming in complex space who extended the basic theorems of linear programming over complex space. In particular, using a variant of the Farkas lemma from real space to complex space, he generalized duality theorems from real linear programming. Since then, linear fractional, nonlinear, and nonlinear fractional complex programming problems were studied by many researchers (see [2-5]).

Minimax problems are encountered in several important contexts. One of the major context is zero sum games, where the objective of the first player is to minimize the amount given to the other player and the objective of the second player is to maximize this amount. Ahmad and Husain [6] established sufficient optimality conditions for a class of nondifferentiable minimax fractional programming problems involving $(F, \alpha, \rho, d)$-convexity. Later on, Jayswal et al. [7] extended the work of Ahmad and Husain [6] to establish sufficient optimality conditions and duality theorems for the nondifferentiable minimax fractional problem under the assumptions of generalized $(F, \alpha, \rho, d)$-convexity. Recently, Jayswal and Kumar [8] established sufficient optimality conditions and duality theorems for a class of nondifferentiable minimax fractional programming problems under the assumptions of $(C, \alpha, \rho, d)$-convexity. Lai et al. [9] established several sufficient optimality conditions for minimax programming in complex spaces under the assumptions of generalized convexity of complex functions. Subsequently, they applied the optimality conditions to formulate parametric dual and derived weak, strong, and strict converse duality theorems.

The first work on fractional programming in complex space appeared in 1970, when Swarup and Sharma [10] generalized the results of Charnes and Cooper [11] to the complex space. Lai and Huang [12] showed that a minimax fractional programming problem is equivalent to a minimax nonfractional parametric problem for a given parameter in complex space and established the necessary and sufficient optimality conditions for nondifferentiable minimax fractional programming problem with complex variables under generalized convexity assumptions.

Recently, Lai and Liu [13] considered a nondifferentiable minimax programming problem in complex space and established the appropriate duality theorems for parametric dual and parameter free dual models. They showed that there is no duality gap between the two dual problems with respect to the primal problem under some generalized convexities of complex functions in the complex programming problem.

In this paper, we focus our study on nondifferentiable minimax programming over complex spaces. The paper is organized as follows. In Section 2, we recall some notations 
and definitions in complex spaces. In Section 3, we establish sufficient optimality conditions under generalized convexity assumptions. Weak, strong, and strict converse duality theorems related to nondifferentiable minimax programming problems in complex spaces for two types of dual models are established in Sections 4 and 5 followed by the conclusion in Section 6 .

\section{Notations and Preliminaries}

We use the following notations that appear in most works on mathematical programming in complex space:

$C^{n}\left(R^{n}\right)=n$-dimensional vector space of complex (real) numbers,

$C^{m \times n}\left(R^{m \times n}\right)=$ the set of $m \times n$ complex (real) matrices, $R_{+}^{n}=\left\{x \in R^{n}, x_{j} \geq 0, j=1,2, \ldots, n\right\}=$ the nonnegative orthant of $R^{n}$,

$A^{H}=\bar{A}^{T}=$ the conjugate transpose of $A=\left[a_{i j}\right]$,

$\langle z, u\rangle=u^{H} z=$ the inner product of $u, z$ in $C^{n}$.

Now, we recall some definitions related to mathematical programming in complex space that are used in the sequel of the paper.

Definition 1 (see [5]). A subset $S \subseteq C^{n}$ is polyhedral cone if there is $k \in N$ and $A \in C^{n \times k}$ such that $S=A R_{+}^{k}=\{A x \mid x \in$ $\left.R_{+}^{k}\right\}$; that is, $S$ is generated by a finite number of vectors (the columns of $A$ ).

Equivalently, $S \subseteq C^{n}$ is said to be a polyhedral cone if it is the intersection of a finite number of closed half-spaces having the origin on the boundary; that is, there is a natural number $p$ and $p$-points $u_{1}, u_{2}, \ldots, u_{p}$ such that

$$
S=\bigcap_{k=1}^{p} D\left(u_{k}\right)=\left\{z \in C^{n} \mid \operatorname{Re}\left\langle z, u_{k}\right\rangle \geq 0, k=1,2, \ldots, p\right\} \text {, }
$$

where $D\left(u_{k}\right), k=1,2, \ldots, p$ are closed half-spaces involving the point $u_{k}$.

Definition 2 (see [5]). If $\emptyset \neq S \subset C^{n}$, then $S^{*}=\left\{y \in C^{n} \mid\right.$ for all $\left.z \in S \Rightarrow \operatorname{Re}\left(y^{H} z\right) \geq 0\right\}$ constitute the dual (polar) of S.

If $\Theta: C^{n} \rightarrow C$ is analytic in a neighbourhood of $z_{0} \in C^{n}$, then $\nabla_{z} \Theta\left(z_{0}\right)=\left[\partial \Theta\left(z_{0}\right) / \partial z_{i}\right], i=1,2, \ldots, n$, is the gradient of function $\Theta$ at $z_{0}$. Similarly, if the complex function $\Theta\left(w^{1}, w^{2}\right)$ is analytic in $2 n$ variables $\left(w^{1}, w^{2}\right)$ and $\left(z_{0}, \bar{z}_{0}\right) \in C^{2 n}$, we define the gradients by

$$
\begin{aligned}
& \nabla_{z} \Theta\left(z_{0}, \bar{z}_{0}\right)=\left[\frac{\partial \Theta\left(z_{0}, \bar{z}_{0}\right)}{\partial w_{j}^{1}}\right], \quad j=1,2, \ldots, n, \\
& \nabla_{\bar{z}} \Theta\left(z_{0}, \bar{z}_{0}\right)=\left[\frac{\partial \Theta\left(z_{0}, \bar{z}_{0}\right)}{\partial w_{j}^{2}}\right], \quad j=1,2, \ldots, n .
\end{aligned}
$$

In this paper, we consider the following complex programming problem:

$$
\begin{array}{ll}
\min _{\zeta \in X} & \sup _{\eta \in Y} \operatorname{Re}\left[f(\zeta, \eta)+\left(z^{H} A z\right)^{1 / 2}\right] \\
\text { subject to } & \zeta \in X=\left\{\zeta=(z, \bar{z}) \in C^{2 n} \mid-h(\zeta) \in S\right\},
\end{array}
$$

where $Y=\left\{\eta=(w, \bar{w}) \mid w \in C^{m}\right\}$ is a compact subset in $C^{2 m}, A \in C^{n \times n}$ is a positive semidefinite Hermitian matrix, $S$ is a polyhedral cone in $C^{p}, f(\cdot, \cdot)$ is continuous, and, for each $\eta \in Y, f(\cdot, \eta): C^{2 n} \rightarrow C$ and $h(\cdot): C^{2 n} \rightarrow C^{p}$ are analytic in $Q=\left\{(z, \bar{z}) \mid z \in C^{n}\right\} \subset C^{2 n}$, where $Q$ is a linear manifold over a real field. In order to have a convex real part for a nonlinear analytic function, the complex functions need to be defined on the linear manifold over $R$; that is, $Q=\left\{\zeta=(z, \bar{z}) \in C^{2 n} \mid\right.$ $\left.z \in C^{n}\right\}$.

Special Cases. (i) If problem (P) is a real programming problem with two variables nondifferentiable minimax problem, it may be expressed as

$$
\begin{array}{ll}
\min & \sup _{y \in Y} f(x, y)+\left(x^{T} B x\right)^{1 / 2} \\
\text { s.t. } & g(x) \leq 0, \quad x \in R^{n},
\end{array}
$$

where $Y$ is compact subset of $R^{l}, f(\cdot, \cdot): R^{n} \times R^{l} \rightarrow R$ and $g(\cdot): R^{n} \rightarrow R^{m}$ are continuously differentiable functions at $x \in R^{n}$, and $B$ is a positive semidefinite symmetric matrix. This problem was studied by Ahmad et al. [14, 15].

(ii) If $Y$ vanishes in $(\mathrm{P})$, then problem $(\mathrm{P})$ reduces to the problem considered by Mond and Craven [16]; that is,

$$
\begin{array}{ll}
\min & \operatorname{Re}\left[f(\zeta)+\left(z^{H} A z\right)^{1 / 2}\right] \\
\text { s.t. } & \zeta \in X=\left\{\zeta \in C^{2 n} \mid-h(\zeta) \in S, \zeta=(z, \bar{z}), z \in C^{n}\right\} .
\end{array}
$$

(iii) If $A=0$, then (P) becomes a differentiable complex minimax programming problem studied by Datta and Bhatia [3]; that is,

$$
\begin{array}{ll}
\min _{\zeta \in X} \sup _{\eta \in Y} \operatorname{Re} f(\zeta, \eta) \\
\text { s.t. } \quad \zeta \in X=\left\{\zeta \in C^{2 n} \mid-h(\zeta) \in S\right\} .
\end{array}
$$

Definition 3. A functional $F: C^{n} \times C^{n} \times C^{n} \rightarrow R$ is said to be sublinear in its third variable, if, for any $z_{1}, z_{2} \in C^{n}$, the following conditions are satisfied:

(i) $F\left(z_{1}, z_{2} ; u_{1}+u_{2}\right) \leq F\left(z_{1}, z_{2} ; u_{1}\right)+F\left(z_{1}, z_{2} ; u_{2}\right)$,

(ii) $F\left(z_{1}, z_{2} ; \alpha u\right)=\alpha F\left(z_{1}, z_{2} ; u\right)$,

for any $\alpha \geq 0$ in $R_{+}$and $u_{1}, u_{2}, u \in C^{n}$. From (ii), it is clear that $F\left(z_{1}, z_{2} ; 0\right)=0$.

Let $F: C^{n} \times C^{n} \times C^{n} \rightarrow R$ be sublinear on the third variable, $\theta: C^{n} \times C^{n} \rightarrow R_{+}$with $\theta\left(z_{1}, z_{2}\right)=0$, if $z_{1}=z_{2}$ and $\alpha: C^{n} \times C^{n} \times \rightarrow R_{+} \backslash\{0\}$. Let $f$ and $h$ be analytic functions and $\rho$ let be a real number. Now, we introduce the following definitions, which are extensions of the definitions given by Lai et al. [9] and Mishra and Rueda [17]. 
Definition 4. The real part $\operatorname{Re}[f]$ of analytic function $f: Q \subset$ $C^{2 n} \rightarrow C$ is said to be $(F, \alpha, \rho, \theta)$-convex (strict $(F, \alpha, \rho, \theta)$ convex) with respect to $R_{+}$on the manifold $Q=\{\zeta=(z, \bar{z}) \mid$ $\left.z \in C^{n}\right\} \subset C^{2 n}$, if, for any $\zeta=(z, \bar{z}), \zeta_{0}=\left(z_{0}, \bar{z}_{0}\right) \in Q$, one has

$$
\begin{aligned}
& \operatorname{Re}\left[f(z, \bar{z})-f\left(z_{0}, \bar{z}_{0}\right)\right] \\
& \geq(>) F\left(z, z_{0} ; \alpha\left(z, z_{0}\right)\right. \\
& \left.\quad \times\left(\overline{\nabla_{z} f\left(z_{0}, \bar{z}_{0}\right)}+\nabla_{\bar{z}} f\left(z_{0}, \bar{z}_{0}\right)\right)\right)+\rho \theta^{2}\left(z, z_{0}\right) .
\end{aligned}
$$

Definition 5. The real part $\operatorname{Re}[f]$ of analytic function $f$ : $Q \subset C^{2 n} \rightarrow C$ is said to be $(F, \alpha, \rho, \theta)$-quasiconvex (strict $(F, \alpha, \rho, \theta)$-quasiconvex) with respect to $R_{+}$on the manifold $Q=\left\{\zeta=(z, \bar{z}) \mid z \in C^{n}\right\} \subset C^{2 n}$, if, for any $\zeta=(z, \bar{z})$, $\zeta_{0}=\left(z_{0}, \bar{z}_{0}\right) \in Q$, one has

$$
\begin{aligned}
& \operatorname{Re}\left[f(z, \bar{z})-f\left(z_{0}, \bar{z}_{0}\right)\right] \\
& \begin{aligned}
\leq(<) 0 \Longrightarrow F\left(z, z_{0} ; \alpha\left(z, z_{0}\right)\right. \\
\left.\quad \times\left(\overline{\nabla_{z} f\left(z_{0}, \bar{z}_{0}\right)}+\nabla_{\bar{z}} f\left(z_{0}, \bar{z}_{0}\right)\right)\right)
\end{aligned} \\
& \quad \leq-\rho \theta^{2}\left(z, z_{0}\right) .
\end{aligned}
$$

Definition 6. The real part $\operatorname{Re}[f]$ of analytic function $f$ : $Q \subset C^{2 n} \rightarrow C$ is said to be $(F, \alpha, \rho, \theta)$-pseudoconvex (strict $(F, \alpha, \rho, \theta)$-pseudoconvex) with respect to $R_{+}$on the manifold $Q=\left\{\zeta=(z, \bar{z}) \mid z \in C^{n}\right\} \subset C^{2 n}$, if, for any $\zeta=(z, \bar{z})$, $\zeta_{0}=\left(z_{0}, \bar{z}_{0}\right) \in Q$, one has

$$
\begin{aligned}
& F\left(z, z_{0} ; \alpha\left(z, z_{0}\right)\left(\overline{\nabla_{z} f\left(z_{0}, \bar{z}_{0}\right)}+\nabla_{\bar{z}} f\left(z_{0}, \bar{z}_{0}\right)\right)\right) \\
& \quad \geq-\rho \theta^{2}\left(z, z_{0}\right) \Longrightarrow \operatorname{Re}\left[f(z, \bar{z})-f\left(z_{0}, \bar{z}_{0}\right)\right] \\
& \quad \geq(>) 0 .
\end{aligned}
$$

Definition 7. The mapping $h: C^{2 n} \rightarrow C^{p}$ is said to be $(F, \alpha, \rho, \theta)$-convex (strict $(F, \alpha, \rho, \theta)$-convex) with respect to the polyhedral cone $S \subset C^{p}$ on the manifold $Q$ if, for any $\mu \in S$ and $\zeta=(z, \bar{z}), \zeta_{0}=\left(z_{0}, \bar{z}_{0}\right) \in Q$, one has

$$
\begin{aligned}
& \operatorname{Re}\langle\mu, h(\zeta)-\left.h\left(\zeta_{0}\right)\right\rangle \\
& \geq(>) F\left(z, z_{0} ; \alpha\left(z, z_{0}\right)\right. \\
&\left.\quad \times\left(\mu^{T} \overline{\nabla_{z} h\left(\zeta_{0}\right)}+\mu^{H} \nabla_{\bar{z}} h\left(\zeta_{0}\right)\right)\right)+\rho \theta^{2}\left(z, z_{0}\right) .
\end{aligned}
$$

Definition 8. The mapping $h: C^{2 n} \rightarrow C^{p}$ is said to be $(F, \alpha, \rho, \theta)$-quasiconvex (strict $(F, \alpha, \rho, \theta)$-quasiconvex) with respect to the polyhedral cone $S \subset C^{p}$ on the manifold $Q$ if, for any $\mu \in S$ and $\zeta=(z, \bar{z}), \zeta_{0}=\left(z_{0}, \bar{z}_{0}\right) \in Q$, one has

$$
\begin{aligned}
& \operatorname{Re}\left\langle\mu, h(\zeta)-h\left(\zeta_{0}\right)\right\rangle \\
& \leq(<) 0 \Longrightarrow F\left(z, z_{0} ; \alpha\left(z, z_{0}\right)\right. \\
& \left.\times\left(\mu^{T} \overline{\nabla_{z} h\left(\zeta_{0}\right)}+\mu^{H} \nabla_{\bar{z}} h\left(\zeta_{0}\right)\right)\right) \\
& \leq-\rho \theta^{2}\left(z, z_{0}\right) \text {. }
\end{aligned}
$$

Definition 9. The mapping $h: C^{2 n} \rightarrow C^{p}$ is said to be $(F, \alpha, \rho, \theta)$-pseudoconvex (strict $(F, \alpha, \rho, \theta)$-pseudoconvex) with respect to the polyhedral cone $S \subset C^{p}$ on the manifold $Q$ if for any $\mu \in S$ and $\zeta=(z, \bar{z}), \zeta_{0}=\left(z_{0}, \bar{z}_{0}\right) \in Q$, one has

$$
\begin{aligned}
& F\left(z, z_{0} ; \alpha\left(z, z_{0}\right)\left(\mu^{T} \overline{\nabla_{z} h\left(\zeta_{0}\right)}+\mu^{H} \nabla_{\bar{z}} h\left(\zeta_{0}\right)\right)\right) \\
& \quad \geq \rho \theta^{2}\left(z, z_{0}\right) \Longrightarrow \operatorname{Re}\left\langle\mu, h(\zeta)-h\left(\zeta_{0}\right)\right\rangle \\
& \quad \geq(>) 0 .
\end{aligned}
$$

Remark 10. In the proofs of theorems, sometimes it may be more convenient to use certain alternative but equivalent forms of the above definitions. Consider the following example.

The real part $\operatorname{Re}[f]$ of analytic function $f: Q \subset C^{2 n} \rightarrow C$ is said to be $(F, \alpha, \rho, \theta)$-pseudoconvex with respect to $R_{+}$on the manifold $Q=\left\{\zeta=(z, \bar{z}) \mid z \in C^{n}\right\} \subset C^{2 n}$, if, for any $\zeta_{0}=\left(z_{0}, \bar{z}_{0}\right) \in \mathrm{Q}$, one has

$$
\begin{aligned}
& \operatorname{Re}\left[f(z, \bar{z})-f\left(z_{0}, \bar{z}_{0}\right)\right] \\
& <0 \Longrightarrow F\left(z, z_{0} ; \alpha\left(z, z_{0}\right)\right. \\
& \left.\quad \times\left(\overline{\nabla_{z} f\left(z_{0}, \bar{z}_{0}\right)}+\nabla_{\bar{z}} f\left(z_{0}, \bar{z}_{0}\right)\right)\right) \\
& \quad<-\rho \theta^{2}\left(z, z_{0}\right) .
\end{aligned}
$$

Remark 11. If we take $\alpha\left(z, z_{0}\right)=1$, then the above definitions reduce to that given by Lai et al. [9]. In addition, if we take $\rho=0$, then we obtain the definitions given by Mishra and Rueda [17].

Let $A \in C^{n \times n}$ and $z, u \in C^{n}$; then Schwarz inequality can be written as

$$
\operatorname{Re}\left(z^{H} A u\right) \leq\left(z^{H} A z\right)^{1 / 2}\left(u^{H} A u\right)^{1 / 2} .
$$

The equality holds if $A z=\lambda A u$ or $z=\lambda u$ for $\lambda \geq 0$.

Definition 12 (see [12]). The problem (P) is said to satisfy the constraint qualification at a point $\zeta_{0}=\left(z_{0}, \bar{z}_{0}\right)$, if, for any nonzero $\mu \in S^{*} \subset C^{p}$,

$$
\operatorname{Re}\left\langle h_{\zeta}^{\prime}\left(\zeta_{0}\right)\left(\zeta-\zeta_{0}\right), \mu\right\rangle \neq 0, \quad \text { for } \zeta \neq \zeta_{0}
$$

In the next section, we recall some notations and discuss necessary and sufficient optimality conditions for problem (P) on the basis of Lai and Liu [18] and Lai and Huang [12]. 


\section{Necessary and Sufficient Conditions}

Let $f(\zeta, \cdot), \zeta=(z, \bar{z}) \in C^{2 n}$ be a continuous function defined on $Y$, where $Y \subset C^{2 m}$ is a specified compact subset in problem (P). Then the supremum $\sup _{\nu \in Y} \operatorname{Re} f(\zeta, \nu)$ will be attained to its maximum in $Y$, and the set

$$
Y(\zeta)=\left\{\eta \in Y \mid \operatorname{Re} f(\zeta, \eta)=\sup _{\nu \in Y} \operatorname{Re} f(\zeta, \nu)\right\}
$$

is then also a compact set in $C^{2 m}$. In particular, if $\zeta=\zeta_{0}=$ $\left(z_{0}, \bar{z}_{0}\right)$ is an optimal solution of problem $(\mathrm{P})$, there exist a positive integer $k$ and finite points $\eta_{i} \in Y\left(\zeta_{0}\right), \lambda_{i}>0, i=$ $1,2, \ldots, k$ with $\sum_{i=1}^{k} \lambda_{i}=1$ such that the Lagrangian function

$$
\phi(\zeta)=\sum_{i=1}^{k} \lambda_{i} f\left(\zeta, \eta_{i}\right)+\langle h(\zeta), \mu\rangle, \quad\left(\mu \neq 0 \text { in } S^{*}\right)
$$

satisfies the Kuhn-Tucker type condition at $\zeta_{0}$. That is,

$$
\begin{gathered}
\left(\sum_{i=1}^{k} \lambda_{i} f_{\zeta}^{\prime}\left(\zeta_{0}, \eta_{i}\right)+\left\langle h_{\zeta}^{\prime}\left(\zeta_{0}\right), \mu\right\rangle\right)\left(\zeta-\zeta_{0}\right)=0 \\
\operatorname{Re}\left\langle h\left(\zeta_{0}\right), \mu\right\rangle=0
\end{gathered}
$$

Equivalent form of expression (15) at $\zeta=\zeta_{0} \in Q$ is

$$
\begin{aligned}
& \sum_{i=1}^{k} \lambda_{i}\left[\overline{\nabla_{z} f\left(\zeta_{0}, \eta_{i}\right)}+\nabla_{\bar{z}} f\left(\zeta_{0}, \eta_{i}\right)\right] \\
& \quad+\left(\mu^{T} \overline{\nabla_{z} h\left(\zeta_{0}\right)}+\mu^{H} \nabla_{\bar{z}} h\left(\zeta_{0}\right)\right)=0 .
\end{aligned}
$$

For the integer $k$, corresponding a vector $\tilde{\eta} \equiv\left(\eta_{1}, \eta_{2}, \ldots, \eta_{k}\right) \in$ $Y\left(\zeta_{0}\right)^{k}$ and $\lambda_{i}>0, i=1,2, \ldots, k$ with $\sum_{i=1}^{k} \lambda_{i}=1$, we define a set as follows:

$$
\begin{aligned}
Z_{\tilde{\eta}}\left(\zeta_{0}\right)= & \left\{\zeta \in C^{2 n} \mid-h_{\zeta}^{\prime}\left(\zeta_{0}\right) \zeta \in S\left(-h\left(\zeta_{0}\right)\right),\right. \\
\zeta & =(z, \bar{z}) \in Q, \\
& \left.\operatorname{Re}\left[\sum_{i=1}^{k} \lambda_{i} f_{\zeta}^{\prime}\left(\zeta_{0}, \eta_{i}\right) \zeta+\langle A z, z\rangle^{1 / 2}\right]<0\right\},
\end{aligned}
$$

where the set $S\left(s_{0}\right)$ is the intersection of closed half-spaces having the point $s_{0} \in S$ on their boundaries.

Theorem 13 (necessary optimality conditions). Let $\zeta_{0}=$ $\left(z_{0}, \bar{z}_{0}\right) \in Q$ be an optimal solution to $(P)$. Suppose that the constraint qualification is satisfied for $(P)$ at $\zeta_{0}$ and $z_{0}^{H} A z_{0}=$ $\left\langle A z_{0}, z_{0}\right\rangle>0$. Then there exist $0 \neq \mu \in S^{*} \subset C^{p}, u \in C^{n}$ and $a$ positive integer $k$ with the following properties:

(i) $\eta_{i} \in Y\left(\zeta_{0}\right), i=1,2, \ldots, k$,

(ii) $\lambda_{i}>0, i=1,2, \ldots, k, \sum_{i=1}^{k} \lambda_{i}=1$, such that $\sum_{i=1}^{k} \lambda_{i} f\left(\zeta, \eta_{i}\right)+\langle h(\zeta), \mu\rangle+\langle A z, z\rangle^{1 / 2}$ satisfies the following conditions:

$$
\begin{gathered}
\sum_{i=1}^{k} \lambda_{i}\left\{\overline{\nabla_{z} f\left(\zeta_{0}, \eta_{i}\right)}+\nabla_{\bar{z}} f\left(\zeta_{0}, \eta_{i}\right)+A u\right\} \\
+\left(\mu^{T} \overline{\nabla_{z} h\left(\zeta_{0}\right)}+\mu^{H} \nabla_{\bar{z}} h\left(\zeta_{0}\right)\right)=0 \\
\operatorname{Re}\left\langle h\left(\zeta_{0}\right), \mu\right\rangle=0 \\
u^{H} A u \leq 1 \\
\left(z_{0}^{H} A z_{0}\right)^{1 / 2}=\operatorname{Re}\left(z_{0}^{H} A u\right) .
\end{gathered}
$$

Theorem 14 (sufficient optimality conditions). Let $\zeta_{0}=$ $\left(z_{0}, \bar{z}_{0}\right) \in Q$ be a feasible solution to $(P)$. Suppose that there exists a positive integer $k, \lambda_{i}>0, \eta_{i} \in Y\left(\zeta_{0}\right), i=1,2, \ldots, k$ with $\sum_{i=1}^{k} \lambda_{i}=1$ and $0 \neq \mu \in S^{*} \subset C^{p}$ satisfying conditions (19)(22). Further, if $\operatorname{Re}\left[\sum_{i=1}^{k} \lambda_{i}\left[f\left(\zeta, \eta_{i}\right)+z^{H} A u\right]\right]$ is $\left(F, \alpha_{1}, \rho_{1}, \theta\right)$ convex with respect to $R_{+}$on $Q, h(\zeta)$ is $\left(F, \alpha_{2}, \rho_{2}, \theta\right)$-convex on $Q$ with respect to the polyhedral cone $S \subset C^{p}$, and $\rho_{1} / \alpha_{1}\left(z, z_{0}\right)+$ $\rho_{2} / \alpha_{2}\left(z, z_{0}\right) \geq 0$, then $\zeta_{0}=\left(z_{0}, \bar{z}_{0}\right)$ is an optimal solution to $(P)$.

Proof. We prove this theorem by contradiction. Suppose that there is a feasible solution $\zeta \in Q$ such that

$$
\begin{aligned}
\sup _{\eta \in Y} \operatorname{Re}\left[f(\zeta, \eta)+\left(z^{H} A z\right)^{1 / 2}\right] \\
\quad<\sup _{\eta \in Y} \operatorname{Re}\left[f\left(\zeta_{0}, \eta\right)+\left(z_{0}^{H} A z_{0}\right)^{1 / 2}\right] .
\end{aligned}
$$

Since $\eta_{i} \in Y\left(\zeta_{0}\right), i=1,2, \ldots, k$, we have

$$
\begin{gathered}
\sup _{\eta \in Y} \operatorname{Re}\left[f\left(\zeta_{0}, \eta\right)+\left(z_{0}^{H} A z_{0}\right)^{1 / 2}\right] \\
=\operatorname{Re}\left[f\left(\zeta_{0}, \eta_{i}\right)+\left(z_{0}^{H} A z_{0}\right)^{1 / 2}\right], \\
\text { for } i=1,2, \ldots, k, \\
\operatorname{Re}\left[f\left(\zeta, \eta_{i}\right)+\left(z^{H} A z\right)^{1 / 2}\right] \\
<\sup _{\eta \in Y} \operatorname{Re}\left[f(\zeta, \eta)+\left(z^{H} A z\right)^{1 / 2}\right], \\
\text { for } i=1,2, \ldots, k .
\end{gathered}
$$

Thus, from the above three inequalities, we obtain

$$
\begin{array}{r}
\operatorname{Re}\left[f\left(\zeta, \eta_{i}\right)+\left(z^{H} A z\right)^{1 / 2}\right] \\
<\operatorname{Re}\left[f\left(\zeta_{0}, \eta_{i}\right)+\left(z_{0}^{H} A z_{0}\right)^{1 / 2}\right] \\
\text { for } i=1,2, \ldots, k .
\end{array}
$$


Using (21) and generalized Schwarz inequality, we get

$$
\begin{aligned}
\operatorname{Re}\left(z^{H} A u\right) & \leq\left(z^{H} A z\right)^{1 / 2}\left(u^{H} A u\right)^{1 / 2} \\
& \leq\left(z^{H} A z\right)^{1 / 2} \\
& =\operatorname{Re}\left[\left(z^{H} A z\right)^{1 / 2}\right]
\end{aligned}
$$

and inequality (22) yields

$$
\operatorname{Re}\left(z_{0}^{H} A u\right)=\operatorname{Re}\left[\left(z_{0}^{H} A z_{0}\right)^{1 / 2}\right] .
$$

Using (26) and (27) in (25), we have

$$
\begin{array}{r}
\operatorname{Re}\left[f\left(\zeta, \eta_{i}\right)+z^{H} A u\right]<\operatorname{Re}\left[f\left(\zeta_{0}, \eta_{i}\right)+z_{0}^{H} A u\right], \\
\text { for } i=1,2, \ldots, k .
\end{array}
$$

Since $\lambda_{i}>0$ and $\sum_{i=1}^{k} \lambda_{i}=1$, we have

$$
\begin{aligned}
& r l \operatorname{Re}\left[\sum_{i=1}^{k} \lambda_{i}\left[f\left(\zeta, \eta_{i}\right)+z^{H} A u\right]-\sum_{i=1}^{k} \lambda_{i}\left[f\left(\zeta_{0}, \eta_{i}\right)+z_{0}^{H} A u\right]\right] \\
& \quad<0 .
\end{aligned}
$$

Since $\operatorname{Re}\left[\sum_{i=1}^{k} \lambda_{i}\left[f\left(\zeta, \eta_{i}\right)+z^{H} A u\right]\right]$ is $\left(F, \alpha_{1}, \rho_{1}, \theta\right)$-convex with respect to $R_{+}$on $Q$, we have

$$
\begin{aligned}
& \operatorname{Re}\left[\sum_{i=1}^{k} \lambda_{i}\left[f\left(\zeta, \eta_{i}\right)+z^{H} A u\right]\right. \\
& \left.\quad-\sum_{i=1}^{k} \lambda_{i}\left[f\left(\zeta_{0}, \eta_{i}\right)+z_{0}^{H} A u\right]\right] \\
& \geq F\left[z, z_{0} ; \alpha_{1}\left(z, z_{0}\right)\right. \\
& \left.\quad \times\left(\sum_{i=1}^{k} \lambda_{i}\left\{\overline{\nabla_{z} f\left(\zeta_{0}, \eta_{i}\right)}+\nabla_{\bar{z}} f\left(\zeta_{0}, \eta_{i}\right)+A u\right\}\right)\right] \\
& +\rho_{1} \theta^{2}\left(z, z_{0}\right) .
\end{aligned}
$$

From (29) and (30), we conclude that

$$
\begin{aligned}
F[z, & z_{0} ; \alpha_{1}\left(z, z_{0}\right) \\
& \left.\times\left(\sum_{i=1}^{k} \lambda_{i}\left\{\overline{\nabla_{z} f\left(\zeta_{0}, \eta_{i}\right)}+\nabla_{\bar{z}} f\left(\zeta_{0}, \eta_{i}\right)+A u\right\}\right)\right] \\
& <-\rho_{1} \theta^{2}\left(z, z_{0}\right),
\end{aligned}
$$

which due to sublinearity of $F$ can be written as

$$
\begin{aligned}
& F\left[z, z_{0} ; \sum_{i=1}^{k} \lambda_{i}\left\{\overline{\nabla_{z} f\left(\zeta_{0}, \eta_{i}\right)}+\nabla_{\bar{z}} f\left(\zeta_{0}, \eta_{i}\right)+A u\right\}\right] \\
& \quad<-\frac{\rho_{1}}{\alpha_{1}\left(z, z_{0}\right)} \theta^{2}\left(z, z_{0}\right) .
\end{aligned}
$$

On the other hand, from the feasibility of $\zeta$ to $(\mathrm{P})$, we have $-h(\zeta) \in S$, or $\operatorname{Re}\langle h(\zeta), \mu\rangle \leq 0$ for $\mu \in S^{*}$, which along with (20) yields

$$
\operatorname{Re}\langle h(\zeta), \mu\rangle \leq 0=\operatorname{Re}\left\langle h\left(\zeta_{0}\right), \mu\right\rangle
$$

Since $h(\zeta)$ is $\left(F, \alpha_{2}, \rho_{2}, \theta\right)$-convex on $Q$ with respect to the polyhedral cone $S \subset C^{p}$, we have

$$
\begin{aligned}
& \operatorname{Re}\langle h(\zeta), \mu\rangle-\operatorname{Re}\left\langle h\left(\zeta_{0}\right), \mu\right\rangle \\
& \geq F\left[z, z_{0} ; \alpha_{2}\left(z, z_{0}\right)\left(\mu^{T} \overline{\nabla_{z} h\left(\zeta_{0}\right)}+\mu^{H} \nabla_{\bar{z}} h\left(\zeta_{0}\right)\right)\right] \\
& \quad+\rho_{2} \theta^{2}\left(z, z_{0}\right) .
\end{aligned}
$$

From (33) and (34), it follows that

$$
\begin{aligned}
& F\left[z, z_{0} ; \alpha_{2}\left(z, z_{0}\right)\left(\mu^{T} \overline{\nabla_{z} h\left(\zeta_{0}\right)}+\mu^{H} \nabla_{\bar{z}} h\left(\zeta_{0}\right)\right)\right] \\
& \quad \leq-\rho_{2} \theta^{2}\left(z, z_{0}\right)
\end{aligned}
$$

which due to sublinearity of $F$ can be written as

$$
F\left[z, z_{0} ; \mu^{T} \overline{\nabla_{z} h\left(\zeta_{0}\right)}+\mu^{H} \nabla_{\bar{z}} h\left(\zeta_{0}\right)\right] \leq-\frac{\rho_{2}}{\alpha_{2}\left(z, z_{0}\right)} \theta^{2}\left(z, z_{0}\right) .
$$

On adding (32) and (36) and using sublinearity of $F$, we get

$$
\begin{gathered}
F\left[z, z_{0} ; \sum_{i=1}^{k} \lambda_{i}\left\{\overline{\nabla_{z} f\left(\zeta_{0}, \eta_{i}\right)}+\nabla_{\bar{z}} f\left(\zeta_{0}, \eta_{i}\right)+A u\right\}\right. \\
\left.+\mu^{T} \overline{\nabla_{z} h\left(\zeta_{0}\right)}+\mu^{H} \nabla_{\bar{z}} h\left(\zeta_{0}\right)\right] \\
<-\left(\frac{\rho_{1}}{\alpha_{1}\left(z, z_{0}\right)}+\frac{\rho_{2}}{\alpha_{2}\left(z, z_{0}\right)}\right) \theta^{2}\left(z, z_{0}\right) .
\end{gathered}
$$

The above inequality, together with the assumption $\rho_{1} / \alpha_{1}\left(z, z_{0}\right)+\rho_{2} / \alpha_{2}\left(z, z_{0}\right) \geq 0$, gives

$$
\begin{gathered}
F\left[z, z_{0} ; \sum_{i=1}^{k} \lambda_{i}\left\{\overline{\nabla_{z} f\left(\zeta_{0}, \eta_{i}\right)}+\nabla_{\bar{z}} f\left(\zeta_{0}, \eta_{i}\right)+A u\right\}\right. \\
\left.+\mu^{T} \overline{\nabla_{z} h\left(\zeta_{0}\right)}+\mu^{H} \nabla_{\bar{z}} h\left(\zeta_{0}\right)\right]<0,
\end{gathered}
$$

which contradicts (19), hence the theorem. 
Theorem 15 (sufficient optimality conditions). Let $\zeta_{0}=$ $\left(z_{0}, \bar{z}_{0}\right) \in Q$ be a feasible solution to $(P)$. Suppose that there exists a positive integer $k, \lambda_{i}>0, \eta_{i} \in Y\left(\zeta_{0}\right), i=1,2, \ldots, k$ with $\sum_{i=1}^{k} \lambda_{i}=1$ and $0 \neq \mu \in S^{*} \subset C^{p}$ satisfying conditions (19)(22). Further, if $\operatorname{Re}\left[\sum_{i=1}^{k} \lambda_{i}\left[f\left(\zeta, \eta_{i}\right)+z^{H} A u\right]\right]$ is $\left(F, \alpha_{1}, \rho_{1}, \theta\right)$ pseudoconvex with respect to $R_{+}$on $Q, h(\zeta)$ is $\left(F, \alpha_{2}, \rho_{2}, \theta\right)$ quasiconvex on $Q$ with respect to the polyhedral cone $S \subset C^{p}$, and $\rho_{1} / \alpha_{1}\left(z, z_{0}\right)+\rho_{2} / \alpha_{2}\left(z, z_{0}\right) \geq 0$, then $\zeta_{0}=\left(z_{0}, \bar{z}_{0}\right)$ is an optimal solution to $(P)$.

Proof. Proceeding as in Theorem 14, we have

$$
\begin{aligned}
\operatorname{Re} & {\left[\sum_{i=1}^{k} \lambda_{i}\left[f\left(\zeta, \eta_{i}\right)+z^{H} A u\right]-\sum_{i=1}^{k} \lambda_{i}\left[f\left(\zeta_{0}, \eta_{i}\right)+z_{0}^{H} A u\right]\right] } \\
& <0
\end{aligned}
$$

which, by $\left(F, \alpha_{1}, \rho_{1}, \theta\right)$-pseudoconvexity of $\operatorname{Re}\left[\sum_{i=1}^{k} \lambda_{i}\left[f\left(\zeta, \eta_{i}\right)+z^{H} A u\right]\right]$ with respect to $R_{+}$on $Q$, yields

$$
\begin{aligned}
& F\left[z, z_{0} ; \alpha_{1}\left(z, z_{0}\right) \sum_{i=1}^{k} \lambda_{i}\left\{\overline{\nabla_{z} f\left(\zeta_{0}, \eta_{i}\right)}+\nabla_{\bar{z}} f\left(\zeta_{0}, \eta_{i}\right)+A u\right\}\right] \\
& \quad<-\rho_{1} \theta^{2}\left(z, z_{0}\right) .
\end{aligned}
$$

Using the sublinearity of $F$, the above inequality can be written as

$$
\begin{aligned}
& F\left[z, z_{0} ; \sum_{i=1}^{k} \lambda_{i}\left\{\overline{\nabla_{z} f\left(\zeta_{0}, \eta_{i}\right)}+\nabla_{\bar{z}} f\left(\zeta_{0}, \eta_{i}\right)+A u\right\}\right] \\
& \quad<-\frac{\rho_{1}}{\alpha_{1}\left(z, z_{0}\right)} \theta^{2}\left(z, z_{0}\right) .
\end{aligned}
$$

On the other hand, from the feasibility of $\zeta$ to $(\mathrm{P})$, we have $-h(\zeta) \in S$, or $\operatorname{Re}\langle h(\zeta), \mu\rangle \leq 0$ for $\mu \in S^{*}$, which along with (20) yields

$$
\operatorname{Re}\langle h(\zeta), \mu\rangle \leq 0=\operatorname{Re}\left\langle h\left(\zeta_{0}\right), \mu\right\rangle
$$

Since $h(\zeta)$ is $\left(F, \alpha_{2}, \rho_{2}, \theta\right)$-quasiconvex on $Q$ with respect to the polyhedral cone $S \subset C^{p}$, the above inequality yields

$$
\begin{aligned}
& F\left[z, z_{0} ; \alpha_{2}\left(z, z_{0}\right)\left(\mu^{T} \overline{\nabla_{z} h\left(\zeta_{0}\right)}+\mu^{H} \nabla_{\bar{z}} h\left(\zeta_{0}\right)\right)\right] \\
& \quad \leq-\rho_{2} \theta^{2}\left(z, z_{0}\right)
\end{aligned}
$$

which due to sublinearity of $F$ can be written as

$$
F\left[z, z_{0} ; \mu^{T} \overline{\nabla_{z} h\left(\zeta_{0}\right)}+\mu^{H} \nabla_{\bar{z}} h\left(\zeta_{0}\right)\right] \leq-\frac{\rho_{2}}{\alpha_{2}\left(z, z_{0}\right)} \theta^{2}\left(z, z_{0}\right) .
$$

On adding (41) and (44) and using sublinearity of $F$, we get

$$
\begin{gathered}
F\left[z, z_{0} ; \sum_{i=1}^{k} \lambda_{i}\left\{\overline{\nabla_{z} f\left(\zeta_{0}, \eta_{i}\right)}+\nabla_{\bar{z}} f\left(\zeta_{0}, \eta_{i}\right)+A u\right\}\right. \\
\left.\quad+\mu^{T} \overline{\nabla_{z} h\left(\zeta_{0}\right)}+\mu^{H} \nabla_{\bar{z}} h\left(\zeta_{0}\right)\right] \\
<-\left(\frac{\rho_{1}}{\alpha_{1}\left(z, z_{0}\right)}+\frac{\rho_{2}}{\alpha_{2}\left(z, z_{0}\right)}\right) \theta^{2}\left(z, z_{0}\right) .
\end{gathered}
$$

The above inequality, together with the assumption $\rho_{1} / \alpha_{1}\left(z, z_{0}\right)+\rho_{2} / \alpha_{2}\left(z, z_{0}\right) \geq 0$, gives

$$
\begin{gathered}
F\left[z, z_{0} ; \sum_{i=1}^{k} \lambda_{i}\left\{\overline{\nabla_{z} f\left(\zeta_{0}, \eta_{i}\right)}+\nabla_{\bar{z}} f\left(\zeta_{0}, \eta_{i}\right)+A u\right\}\right. \\
\left.+\mu^{T} \overline{\nabla_{z} h\left(\zeta_{0}\right)}+\mu^{H} \nabla_{\bar{z}} h\left(\zeta_{0}\right)\right]<0,
\end{gathered}
$$

which contradicts (19), hence the theorem.

Theorem 16 (sufficient optimality conditions). Let $\zeta_{0}=$ $\left(z_{0}, \bar{z}_{0}\right) \in Q$ be a feasible solution to $(P)$. Suppose that there exists a positive integer $k, \lambda_{i}>0, \eta_{i} \in Y\left(\zeta_{0}\right), i=1,2, \ldots, k$ with $\sum_{i=1}^{k} \lambda_{i}=1$ and $0 \neq \mu \in S^{*} \subset C^{p}$ satisfying conditions (19)(22). Further, if $\operatorname{Re}\left[\sum_{i=1}^{k} \lambda_{i}\left[f\left(\zeta, \eta_{i}\right)+z^{H} A u\right]\right]$ is $\left(F, \alpha_{1}, \rho_{1}, \theta\right)$ quasiconvex with respect to $R_{+}$on $Q, h(\zeta)$ is strict $\left(F, \alpha_{2}, \rho_{2}, \theta\right)$ pseudoconvex on $Q$ with respect to the polyhedral cone $S \subset C^{p}$, and $\rho_{1} / \alpha_{1}\left(z, z_{0}\right)+\rho_{2} / \alpha_{2}\left(z, z_{0}\right) \geq 0$, then $\zeta_{0}=\left(z_{0}, \bar{z}_{0}\right)$ is an optimal solution to $(P)$.

Proof. The proof follows on the similar lines of Theorem 15.

\section{Parametric Duality}

We adopt the following notations in order to simplify the formulation of dual:

$$
\begin{gathered}
K(\xi)=\left\{(k, \tilde{\lambda}, \tilde{\eta}) \in N \times R_{+}^{k} \times C^{2 m k} \mid\right. \\
\tilde{\lambda}=\left(\lambda_{1}, \lambda_{2}, \ldots, \lambda_{k}\right) \quad \text { with } \sum_{i=1}^{k} \lambda_{i}=1, \\
\tilde{\eta}=\left(\eta_{1}, \eta_{2}, \ldots, \eta_{k}\right) \\
\text { with } \left.\eta_{i} \in Y(\xi), i=1,2, \ldots, k\right\},
\end{gathered}
$$

for $\xi=(u, \bar{u}) \in Q \subset C^{2 n}$.

Now, we formulate a parametric dual problem (D1) with respect to the complex minimax programming problem $(\mathrm{P})$ as follows:

$$
\max _{(k, \tilde{\lambda}, \tilde{\eta}) \in K(\xi)} \sup _{(\xi, \mu, w, t) \in X(k, \tilde{\lambda}, \tilde{\eta})} t,
$$


where $X(k, \tilde{\lambda}, \widetilde{\eta})$ denotes the set of all $(\xi, \mu, w, t) \in C^{2 n} \times C^{p} \times$ $C^{n} \times R$ to satisfy the following conditions:

$$
\begin{gathered}
\sum_{i=1}^{k} \lambda_{i}\left[\overline{\nabla_{z} f\left(\xi, \eta_{i}\right)}+\nabla_{\bar{z}} f\left(\xi, \eta_{i}\right)+A w\right] \\
+\mu^{T} \overline{\nabla_{z} h(\xi)}+\mu^{H} \nabla_{\bar{z}} h(\xi)=0, \\
\sum_{i=1}^{k} \lambda_{i}\left\{\operatorname{Re} f\left(\xi, \eta_{i}\right)+\left(u^{H} A u\right)^{1 / 2}-t\right\} \geq 0, \\
\operatorname{Re}\langle h(\xi), \mu\rangle \geq 0, \\
w^{H} A w \leq 1, \\
\left(u^{H} A u\right)^{1 / 2}=\operatorname{Re}\left(u^{H} A w\right), \\
0 \neq \mu \in S^{*} .
\end{gathered}
$$

If, for a triplet $(k, \widetilde{\lambda}, \widetilde{\eta}) \in K(\xi)$, the set $X(k, \widetilde{\lambda}, \widetilde{\eta})=\emptyset$, then we define the supremum over $X(k, \widetilde{\lambda}, \widetilde{\eta})$ to be $-\infty$ for nonexecption in the formulation of (D1).

Theorem 17 (weak duality). Let $\zeta=(z, \bar{z})$ and $(k, \widetilde{\lambda}, \widetilde{\eta}, \xi, \mu, w, t)$ be feasible solutions to $(P)$ and $(D 1)$, respectively. Further, if $\operatorname{Re}\left[\sum_{i=1}^{k} \lambda_{i}\left[f\left(\zeta, \eta_{i}\right)+z^{H} A w\right]\right]$ is $\left(F, \alpha_{1}, \rho_{1}, \theta\right)$-pseudoconvex with respect to $R_{+}$on $Q, h(\zeta)$ is $\left(F, \alpha_{2}, \rho_{2}, \theta\right)$-quasiconvex on $Q$ with respect to the polyhedral cone $S \subset C^{p}$, and $\rho_{1} / \alpha_{1}(z, u)+\rho_{2} / \alpha_{2}(z, u) \geq 0$, Then

$$
\sup _{\tilde{\eta} \in Y} \operatorname{Re}\left[f(\zeta, \widetilde{\eta})+\left(z^{H} A z\right)^{1 / 2}\right] \geq t .
$$

Proof. Suppose, on the contrary, that

$$
\sup _{\tilde{\eta} \in Y} \operatorname{Re}\left[f(\zeta, \widetilde{\eta})+\left(z^{H} A z\right)^{1 / 2}\right]<t .
$$

By compactness of $Y(\xi) \subset Y$ in $C^{p}, \xi \in(u, \bar{u}) \in Q$, there exist an integer $k>0$ and finite points $\eta_{i} \in Y(\xi), \lambda_{i}>0$, $i=1,2, \ldots, k$ with $\sum_{i=1}^{k} \lambda_{i}=1$ such that (49) holds. From (49) and (55), we have

$$
\begin{gathered}
\operatorname{Re}\left[\sum_{i=1}^{k} \lambda_{i}\left[f\left(\zeta, \eta_{i}\right)+\left(z^{H} A z\right)^{1 / 2}\right]\right]<\sum_{i=1}^{k} \lambda_{i} t \\
\leq \sum_{i=1}^{k} \lambda_{i} \operatorname{Re}\left[f\left(\xi, \eta_{i}\right)+\left(u^{H} A u\right)^{1 / 2}\right] .
\end{gathered}
$$

From (51) and the generalized Schwarz inequality, we have

$$
\operatorname{Re}\left(z^{H} A w\right) \leq\left(z^{H} A z\right)^{1 / 2}\left(w^{H} A w\right)^{1 / 2} \leq\left(z^{H} A z\right)^{1 / 2} .
$$

Using (52) and (57) in (56), we get

$$
\begin{aligned}
\operatorname{Re} & {\left[\sum_{i=1}^{k} \lambda_{i}\left[f\left(\zeta, \eta_{i}\right)+z^{H} A w\right]\right] } \\
< & \operatorname{Re}\left[\sum_{i=1}^{k} \lambda_{i}\left[f\left(\xi, \eta_{i}\right)+u^{H} A w\right]\right] .
\end{aligned}
$$

Since $\operatorname{Re}\left[\sum_{i=1}^{k} \lambda_{i}\left[f\left(\zeta, \eta_{i}\right)+z^{H} A w\right]\right]$ is $\left(F, \alpha_{1}, \rho_{1}, \theta\right)$ pseudoconvex with respect to $R_{+}$on $Q$, the above inequality implies that

$$
\begin{aligned}
& F\left[z, u ; \alpha_{1}(z, u) \sum_{i=1}^{k} \lambda_{i}\left\{\overline{\nabla_{z} f\left(\xi, \eta_{i}\right)}+\nabla_{\bar{z}} f\left(\xi, \eta_{i}\right)+A w\right\}\right] \\
& \quad<-\rho_{1} \theta^{2}(z, u),
\end{aligned}
$$

which due to sublinearity of $F$ can be written as

$$
\begin{aligned}
& F\left[z, u ; \sum_{i=1}^{k} \lambda_{i}\left\{\overline{\nabla_{z} f\left(\xi, \eta_{i}\right)}+\nabla_{\bar{z}} f\left(\xi, \eta_{i}\right)+A w\right\}\right] \\
& <-\frac{\rho_{1}}{\alpha_{1}(z, u)} \theta^{2}(z, u) .
\end{aligned}
$$

By the feasibility of $\zeta=(z, \bar{z})$ to $(\mathrm{P})$, we have $-h(\zeta) \in S$, or $\operatorname{Re}\langle h(\zeta), \mu\rangle \leq 0$, for $\mu \in S^{*}$, which along with (50) yields

$$
\operatorname{Re}\langle h(\zeta), \mu\rangle \leq 0 \leq \operatorname{Re}\langle h(\xi), \mu\rangle .
$$

The above inequality, together with the $\left(F, \alpha_{2}, \rho_{2}, \theta\right)$ quasiconvexity of $h(\zeta)$ on $Q$ with respect to the polyhedral cone $S \subset C^{p}$, implies

$$
\begin{aligned}
& F\left[z, u ; \alpha_{2}(z, u)\left(\mu^{T} \overline{\nabla_{z} h(\xi)}+\mu^{H} \nabla_{\bar{z}} h(\xi)\right)\right] \\
& \quad \leq-\rho_{2} \theta^{2}(z, u),
\end{aligned}
$$

which due to sublinearity of $F$ can be written as

$$
\begin{gathered}
F\left[z, u ; \mu^{T} \overline{\nabla_{z} h(\xi)}+\mu^{H} \nabla_{\bar{z}} h(\xi)\right] \\
\leq-\frac{\rho_{2}}{\alpha_{2}(z, u)} \theta^{2}(z, u) .
\end{gathered}
$$

On adding (60) and (63) and using sublinearity of $F$, we get

$$
\begin{gathered}
F\left[z, u ; \sum_{i=1}^{k} \lambda_{i}\left\{\overline{\nabla_{z} f\left(\xi, \eta_{i}\right)}+\nabla_{\bar{z}} f\left(\xi, \eta_{i}\right)+A w\right\}\right. \\
\left.+\mu^{T} \overline{\nabla_{z} h(\xi)}+\mu^{H} \nabla_{\bar{z}} h(\xi)\right] \\
<-\left(\frac{\rho_{1}}{\alpha_{1}(z, u)}+\frac{\rho_{2}}{\alpha_{2}(z, u)}\right) \theta^{2}(z, u) .
\end{gathered}
$$

From the assumption $\rho_{1} / \alpha_{1}(z, u)+\rho_{2} / \alpha_{2}(z, u) \geq 0$, the above inequality yields

$$
\begin{gathered}
F\left[z, u ; \sum_{i=1}^{k} \lambda_{i}\left\{\overline{\nabla_{z} f\left(\xi, \eta_{i}\right)}+\nabla_{\bar{z}} f\left(\xi, \eta_{i}\right)+A w\right\}\right. \\
\left.+\mu^{T} \overline{\nabla_{z} h(\xi)}+\mu^{H} \nabla_{\bar{z}} h(\xi)\right]<0,
\end{gathered}
$$

which contradicts (48), hence the theorem. 
Theorem 18 (weak duality). Let $\zeta=(z, \bar{z})$ and $(k, \widetilde{\lambda}, \widetilde{\eta}, \xi, \mu, w, t)$ be feasible solutions to $(P)$ and $(D 1)$, respectively. Further, if $\operatorname{Re}\left[\sum_{i=1}^{k} \lambda_{i}\left[f\left(\zeta, \eta_{i}\right)+z^{H} A w\right]\right]$ is $\left(F, \alpha_{1}, \rho_{1}, \theta\right)$-quasiconvex with respect to $R_{+}$on $Q, h(\zeta)$ is $\left(F, \alpha_{2}, \rho_{2}, \theta\right)$-pseudoconvex on $Q$ with respect to the polyhedral cone $S \subset C^{p}$, and $\rho_{1} / \alpha_{1}(z, u)+\rho_{2} / \alpha_{2}(z, u) \geq 0$, then

$$
\sup _{\tilde{\eta} \in Y} \operatorname{Re}\left[f(\zeta, \widetilde{\eta})+\left(z^{H} A z\right)^{1 / 2}\right] \geq t
$$

Proof. The proof follows the same lines as in Theorem 17.

Theorem 19 (strong duality). Let $\zeta_{0}=\left(z_{0}, \bar{z}_{0}\right)$ be an optimal solution to the problem $(P)$ at which a constraint qualification is satisfied. Then there exist $(k, \tilde{\lambda}, \widetilde{\eta}) \in K\left(\zeta_{0}\right)$ and $\left(\zeta_{0}, \mu, w, t\right) \in$ $X(k, \tilde{\lambda}, \widetilde{\eta})$ such that $\left(k, \tilde{\lambda}, \widetilde{\eta}, \zeta_{0}, \mu, w, t\right)$ is a feasible solution to the dual problem (D1). If the hypotheses of Theorem 17 or 18 are satisfied, then $\left(k, \tilde{\lambda}, \widetilde{\eta}, \zeta_{0}, \mu, w, t\right)$ is optimal to $(D 1)$, and the two problems $(P)$ and $(D 1)$ have the same optimal values.

Proof. The proof follows along the lines of Theorem 6 (Lai and Liu [13]).

Theorem 20 (strict converse duality). Let $\hat{\zeta}$ and $(\widehat{k}, \hat{\tilde{\lambda}}, \hat{\tilde{\eta}}, \widehat{\xi}, \widehat{\mu}, \widehat{w}, \widehat{t})$ be optimal solutions to $(P)$ and $(D 1)$, respectively, and assume that the assumptions of Theorem 19 are satisfied. Further, assume that the following conditions are satisfied:

(i) $\operatorname{Re} \sum_{i=1}^{\widehat{k}} \widehat{\lambda}_{i}\left[f\left(\widehat{\zeta}, \widehat{\eta}_{i}\right)+\widehat{u}^{H} A \widehat{w}\right]$ is strict $\left(F, \alpha_{1}, \rho_{1}, \theta\right)-$ pseudoconvex with respect to $R_{+}$on $Q$ and $h(\widehat{\zeta})$ is $\left(F, \alpha_{2}, \rho_{2}, \theta\right)$-quasiconvex on $Q$ with respect to the polyhedral cone $S \subset C^{p}$;

(ii) $\rho_{1} / \alpha_{1}(\widehat{z}, \widehat{u})+\rho_{2} / \alpha_{2}(\widehat{z}, \widehat{u}) \geq 0$.

Then $\widehat{\zeta}=\widehat{\xi}$; that is, $\widehat{\zeta}$ is optimal solution to (D1).

Proof. On the contrary, suppose that $(\widehat{z}, \overline{\bar{z}})=\widehat{\zeta} \neq \bar{\xi}=(\widehat{u}, \overline{\hat{u}})$.

On applying Theorem 19, we know that

$$
\widehat{t}=\sup _{\hat{\bar{\eta}} \in Y} \operatorname{Re}\left[f(\widehat{\zeta}, \widehat{\tilde{\eta}})+\left(\hat{z}^{H} A \hat{z}\right)^{1 / 2}\right] .
$$

From the feasibility of $\widehat{\zeta} \in Q$ to $(\mathrm{P}), \widehat{\mu} \in S^{*}$ and (50), we have

$$
\operatorname{Re}\langle h(\widehat{\zeta}), \widehat{\mu}\rangle \leq 0 \leq \operatorname{Re}\langle h(\widehat{\xi}), \widehat{\mu}\rangle .
$$

Since $h(\widehat{\zeta})$ is $\left(F, \alpha_{2}, \rho_{2}, \theta\right)$-quasiconvex on $Q$ with respect to the polyhedral cone $\operatorname{Sin} C^{p}$, the above inequality yields

$$
\begin{aligned}
& F\left[\widehat{z}, \widehat{u} ; \alpha_{2}(\widehat{z}, \widehat{u})\left(\widehat{\mu}^{T} \overline{\nabla_{z} h(\widehat{\xi})}+\widehat{\mu}^{H} \nabla_{\bar{z}} h(\widehat{\xi})\right)\right] \\
& \quad \leq-\rho_{2} \theta^{2}(\widehat{z}, \widehat{u}),
\end{aligned}
$$

which by sublinearity of $F$ implies

$$
\begin{gathered}
F\left[\widehat{z}, \widehat{u} ;\left(\widehat{\mu}^{T} \overline{\nabla_{z} h(\widehat{\xi})}+\widehat{\mu}^{H} \nabla_{\bar{z}} h(\widehat{\xi})\right)\right] \\
\quad \leq-\frac{\rho_{2}}{\alpha_{2}(\widehat{z}, \widehat{u})} \theta^{2}(\widehat{z}, \widehat{u}) .
\end{gathered}
$$

By (48) and the sublinearity of $F$, we have

$$
\begin{gathered}
F\left[\widehat{z}, \widehat{u} ; \sum_{i=1}^{\widehat{k}} \widehat{\lambda}_{i}\left[\overline{\nabla_{z} f\left(\widehat{\xi}, \hat{\eta}_{i}\right)}+\nabla_{\bar{z}} f\left(\widehat{\xi}, \widehat{\eta}_{i}\right)+A \widehat{w}\right]\right] \\
+F\left[\widehat{z}, \widehat{u} ;\left(\hat{\mu}^{T} \overline{\nabla_{z} h(\widehat{\xi})}+\widehat{\mu}^{H} \nabla_{\bar{z}} h(\widehat{\xi})\right)\right] \\
\geq F\left[\widehat{z}, \widehat{u} ; \sum_{i=1}^{\widehat{k}} \hat{\lambda}_{i}\left[\overline{\nabla_{z} f\left(\widehat{\xi}, \hat{\eta}_{i}\right)}+\nabla_{\bar{z}} f\left(\widehat{\xi}, \widehat{\eta}_{i}\right)+A \widehat{w}\right]\right. \\
\left.+\widehat{\mu}^{T} \overline{\nabla_{z} h(\widehat{\xi})}+\widehat{\mu}^{H} \nabla_{\bar{z}} h(\widehat{\xi})\right]=0 .
\end{gathered}
$$

The above inequality, together with (70) and $\rho_{1} / \alpha_{1}(\widehat{z}, \widehat{u})+$ $\rho_{2} / \alpha_{2}(\widehat{z}, \widehat{u}) \geq 0$, gives

$$
\begin{aligned}
& F\left[\widehat{z}, \widehat{u} ; \sum_{i=1}^{\widehat{k}} \widehat{\lambda}_{i}\left[\overline{\nabla_{z} f\left(\widehat{\xi}, \widehat{\eta}_{i}\right)}+\nabla_{\bar{z}} f\left(\widehat{\xi}, \widehat{\eta}_{i}\right)+A \widehat{w}\right]\right] \\
& \quad \geq-F\left[\widehat{z}, \widehat{u} ;\left(\widehat{\mu}^{T} \overline{\nabla_{z} h(\widehat{\xi})}+\widehat{\mu}^{H} \nabla_{\bar{z}} h(\widehat{\xi})\right)\right] \\
& \quad \geq \frac{\rho_{2}}{\alpha_{2}(\widehat{z}, \widehat{u})} \theta^{2}(\widehat{z}, \widehat{u}) \\
& \geq-\frac{\rho_{1}}{\alpha_{1}(\widehat{z}, \widehat{u})} \theta^{2}(\widehat{z}, \widehat{u}) .
\end{aligned}
$$

That is,

$$
\begin{aligned}
& F\left[\widehat{z}, \widehat{u} ; \sum_{i=1}^{\widehat{k}} \widehat{\lambda}_{i}\left[\overline{\nabla_{z} f\left(\widehat{\xi}, \widehat{\eta}_{i}\right)}+\nabla_{\bar{z}} f\left(\widehat{\xi}, \widehat{\eta}_{i}\right)+A \widehat{w}\right]\right] \\
& \geq-\frac{\rho_{1}}{\alpha_{1}(\widehat{z}, \widehat{u})} \theta^{2}(\widehat{z}, \widehat{u}),
\end{aligned}
$$

which by sublinearity of $F$ implies

$$
\begin{aligned}
& F\left[\widehat{z}, \widehat{u} ; \alpha_{1}(\widehat{z}, \widehat{u}) \sum_{i=1}^{\widehat{k}} \widehat{\lambda}_{i}\left[\overline{\nabla_{z} f\left(\widehat{\xi}, \widehat{\eta}_{i}\right)}+\nabla_{\bar{z}} f\left(\widehat{\xi}, \widehat{\eta}_{i}\right)+A \widehat{w}\right]\right] \\
& \quad \geq-\rho_{1} \theta^{2}(\widehat{z}, \widehat{u}) .
\end{aligned}
$$

Since $\operatorname{Re} \sum_{i=1}^{\widehat{k}} \widehat{\lambda}_{i}\left[f\left(\widehat{\zeta}, \widehat{\eta}_{i}\right)+\widehat{u}^{H} A \widehat{w}\right]$ is strict $\left(F, \alpha_{1}, \rho_{1}, \theta\right)$ pseudoconvex with respect to $R_{+}$on $Q$, the above inequality implies that

$$
\begin{gathered}
\operatorname{Re}\left[\sum_{i=1}^{\widehat{k}} \widehat{\lambda}_{i}\left[f\left(\widehat{\zeta}, \widehat{\eta}_{i}\right)+\widehat{z}^{H} A \widehat{w}\right]\right] \\
>\operatorname{Re}\left[\sum_{i=1}^{\hat{k}} \widehat{\lambda}_{i}\left[f\left(\widehat{\xi}, \widehat{\eta}_{i}\right)+\widehat{u}^{H} A \widehat{w}\right]\right] .
\end{gathered}
$$


From (51), (52), and the generalized Schwarz inequality, we have

$$
\operatorname{Re}\left(\widehat{z}^{H} A \widehat{w}\right) \leq\left(\widehat{z}^{H} A \widehat{z}\right)^{1 / 2}, \quad \operatorname{Re}\left(\widehat{u}^{H} A \widehat{w}\right)=\left(\widehat{u}^{H} A \widehat{u}\right)^{1 / 2},
$$

which on substituting in (75) and by using (49), we obtain

$$
\begin{aligned}
& \operatorname{Re}\left[\sum_{i=1}^{\widehat{k}} \widehat{\lambda}_{i}\left[f\left(\widehat{\zeta}, \widehat{\eta}_{i}\right)+\left(\widehat{z}^{H} A \widehat{z}\right)^{1 / 2}\right]\right] \\
& >\operatorname{Re}\left[\sum_{i=1}^{\hat{k}} \hat{\lambda}_{i}\left[f\left(\widehat{\xi}, \widehat{\eta}_{i}\right)+\left(\widehat{u}^{H} A \widehat{u}\right)^{1 / 2}\right]\right] \\
& \geq \sum_{i=1}^{\hat{k}} \hat{\lambda}_{i} \widehat{t}
\end{aligned}
$$

Consequently, there exist certain $i_{0}$ which satisfy

$$
\operatorname{Re}\left[f\left(\widehat{\zeta}, \widehat{\eta}_{i_{0}}\right)+\left(\widehat{z}^{H} A \widehat{z}\right)^{1 / 2}\right]>\widehat{t}
$$

Hence,

$$
\begin{aligned}
& \sup _{\widehat{\tilde{\eta}} \in Y} \operatorname{Re}\left[f(\widehat{\zeta}, \widehat{\tilde{\eta}})+\left(\widehat{z}^{H} A \widehat{z}\right)^{1 / 2}\right] \\
& \quad \geq \operatorname{Re}\left[f\left(\widehat{\zeta}, \widehat{\eta}_{i_{0}}\right)+\left(\widehat{z}^{H} A \widehat{z}\right)^{1 / 2}\right]>\widehat{t},
\end{aligned}
$$

which contradicts (67), hence the theorem.

\section{Parameter Free Duality}

Making use of the optimality conditions, we show that the following formation is a dual (D2) to the complex programming problem $(\mathrm{P})$ :

$$
\max _{(k, \tilde{\lambda}, \tilde{\eta}) \in K(\xi)} \sup _{(\xi, \mu, w) \in X(k, \tilde{\lambda}, \tilde{\eta})} \operatorname{Re}\left[f(\xi, \widetilde{\eta})+\left(u^{H} A u\right)^{1 / 2}\right],
$$

where $X(k, \tilde{\lambda}, \tilde{\eta})$ denotes the set of all $(\xi, \mu, w) \in C^{2 n} \times C^{p} \times C^{n}$ to satisfy the following conditions:

$$
\begin{gathered}
\sum_{i=1}^{k} \lambda_{i}\left[\overline{\nabla_{z} f\left(\xi, \eta_{i}\right)}+\nabla_{\bar{z}} f\left(\xi, \eta_{i}\right)+A w\right] \\
+\mu^{T} \overline{\nabla_{z} h(\xi)}+\mu^{H} \nabla_{\bar{z}} h(\xi)=0, \\
\operatorname{Re}\langle h(\xi), \mu\rangle \geq 0, \\
w^{H} A w \leq 1, \\
\left(u^{H} A u\right)^{1 / 2}=\operatorname{Re}\left(u^{H} A w\right) .
\end{gathered}
$$

If, for a triplet $(k, \tilde{\lambda}, \tilde{\eta}) \in K(\xi)$, the set $X(k, \tilde{\lambda}, \widetilde{\eta})=\emptyset$, then we define the supremum over $X(k, \widetilde{\lambda}, \widetilde{\eta})$ to be $-\infty$ for nonexception in the formulation of (D2).
Now, we establish appropriate duality theorems and prove that optimal values of (P) and (D2) are equal under the assumption of generalized convexity in order to show that the problems (P) and (D2) have no duality gap.

Theorem 21 (weak duality). Let $\zeta=(z, \bar{z})$ and $(k, \tilde{\lambda}, \tilde{\eta}, \xi, \mu, w)$ be feasible solutions to $(P)$ and $(D 2)$, respectively. Further, if $\operatorname{Re}\left[\sum_{i=1}^{k} \lambda_{i}\left[f\left(\zeta, \eta_{i}\right)+z^{H} A w\right]\right]$ is $\left(F, \alpha_{1}, \rho_{1}, \theta\right)$-pseudoconvex with respect to $R_{+}$on $Q, h(\zeta)$ is $\left(F, \alpha_{2}, \rho_{2}, \theta\right)$-quasiconvex on $Q$ with respect to the polyhedral cone $S \subset C^{p}$, and $\rho_{1} / \alpha_{1}(z, u)+$ $\rho_{2} / \alpha_{2}(z, u) \geq 0$, then

$$
\begin{aligned}
& \sup _{\tilde{\eta} \in Y} \operatorname{Re}\left[f(\zeta, \widetilde{\eta})+\left(z^{H} A z\right)^{1 / 2}\right] \\
& \quad \geq \sup _{\tilde{\eta} \in Y} \operatorname{Re}\left[f(\xi, \tilde{\eta})+\left(u^{H} A u\right)^{1 / 2}\right] .
\end{aligned}
$$

Proof. On the contrary, we suppose that

$$
\begin{aligned}
& \sup _{\tilde{\eta} \in Y} \operatorname{Re}\left[f(\zeta, \widetilde{\eta})+\left(z^{H} A z\right)^{1 / 2}\right] \\
& \quad<\sup _{\tilde{\eta} \in Y} \operatorname{Re}\left[f(\xi, \widetilde{\eta})+\left(u^{H} A u\right)^{1 / 2}\right] .
\end{aligned}
$$

Since $\eta_{i} \in Y(\xi) \subset Y, i=1,2, \ldots, k$, we have

$$
\begin{aligned}
& \sup _{\tilde{\eta} \in Y} \operatorname{Re}\left[f(\xi, \tilde{\eta})+\left(u^{H} A u\right)^{1 / 2}\right] \\
& \quad=\operatorname{Re}\left[f\left(\xi, \eta_{i}\right)+\left(u^{H} A u\right)^{1 / 2}\right], \quad i=1,2, \ldots, k, \\
& \operatorname{Re}\left[f\left(\zeta, \eta_{i}\right)+\left(z^{H} A z\right)^{1 / 2}\right] \\
& \quad \leq \sup _{\tilde{\eta} \in Y} \operatorname{Re}\left[f(\zeta, \tilde{\eta})+\left(z^{H} A z\right)^{1 / 2}\right], \quad i=1,2, \ldots, k .
\end{aligned}
$$

Then the above three inequalities give

$$
\begin{aligned}
\operatorname{Re} & {\left[f\left(\zeta, \eta_{i}\right)+\left(z^{H} A z\right)^{1 / 2}\right] } \\
& <\operatorname{Re}\left[f\left(\xi, \eta_{i}\right)+\left(u^{H} A u\right)^{1 / 2}\right], \quad i=1,2, \ldots, k .
\end{aligned}
$$

From (82), (83), (88), and the generalized Schwarz inequality, we have

$$
\begin{aligned}
& \operatorname{Re} {\left[f\left(\zeta, \eta_{i}\right)+\left(z^{H} A w\right)\right] } \\
&<\operatorname{Re}\left[f\left(\xi, \eta_{i}\right)+\left(u^{H} A w\right)\right], \quad i=1,2, \ldots, k .
\end{aligned}
$$

As $\lambda_{i}>0, i=1,2, \ldots, k$ and $\sum_{i=1}^{k} \lambda_{i}=1$, we have

$$
\begin{aligned}
\operatorname{Re} & {\left[\sum_{i=1}^{k} \lambda_{i}\left[f\left(\zeta, \eta_{i}\right)+\left(z^{H} A w\right)\right]\right] } \\
& -\operatorname{Re}\left[\sum_{i=1}^{k} \lambda_{i}\left[f\left(\xi, \eta_{i}\right)+\left(u^{H} A w\right)\right]\right]<0 .
\end{aligned}
$$


Since $\operatorname{Re}\left[\sum_{i=1}^{k} \lambda_{i}\left[f\left(\zeta, \eta_{i}\right)+z^{H} A w\right]\right]$ is $\left(F, \alpha_{1}, \rho_{1}, \theta\right)$ pseudoconvex with respect to $R_{+}$on $Q$, the above inequality implies that

$$
\begin{aligned}
& F\left[z, u ; \alpha_{1}(z, u)\right. \\
& \left.\quad \times \sum_{i=1}^{k} \lambda_{i}\left\{\overline{\nabla_{z} f\left(\xi, \eta_{i}\right)}+\nabla_{\bar{z}} f\left(\xi, \eta_{i}\right)+A w\right\}\right] \\
& \quad<-\rho_{1} \theta^{2}(z, u),
\end{aligned}
$$

which by sublinearity of $F$ becomes

$$
\begin{aligned}
& F\left[z, u ; \sum_{i=1}^{k} \lambda_{i}\left\{\overline{\nabla_{z} f\left(\xi, \eta_{i}\right)}+\nabla_{\bar{z}} f\left(\xi, \eta_{i}\right)+A w\right\}\right] \\
& \quad<-\frac{\rho_{1}}{\alpha_{1}(z, u)} \theta^{2}(z, u) .
\end{aligned}
$$

By the feasibility of $\zeta=(z, \bar{z})$ to $(\mathrm{P}), 0 \neq \mu \in S^{*}$, and the inequality (81), we obtain

$$
\operatorname{Re}\langle h(\zeta), \mu\rangle \leq 0 \leq \operatorname{Re}\langle h(\xi), \mu\rangle .
$$

The above inequality together with the $\left(F, \alpha_{2}, \rho_{2}, \theta\right)$ quasiconvexity of $h(\zeta)$ on $Q$ with respect to the polyhedral cone $S \subset C^{p}$ implies

$$
\begin{aligned}
& F\left[z, u ; \alpha_{2}(z, u)\left(\mu^{T} \overline{\nabla_{z} h(\xi)}+\mu^{H} \nabla_{\bar{z}} h(\xi)\right)\right] \\
& \quad \leq-\rho_{2} \theta^{2}(z, u)
\end{aligned}
$$

which by sublinearity of $F$ becomes

$$
\begin{gathered}
F\left[z, u ; \mu^{T} \overline{\nabla_{z} h(\xi)}+\mu^{H} \nabla_{\bar{z}} h(\xi)\right] \\
\leq-\frac{\rho_{2}}{\alpha_{2}(z, u)} \theta^{2}(z, u) .
\end{gathered}
$$

On adding (92) and (95) and using the sublinearity of $F$, we get

$$
\begin{gathered}
F\left[z, u ; \sum_{i=1}^{k} \lambda_{i}\left\{\overline{\nabla_{z} f\left(\xi, \eta_{i}\right)}+\nabla_{\bar{z}} f\left(\xi, \eta_{i}\right)+A w\right\}\right. \\
\left.+\mu^{T} \overline{\nabla_{z} h(\xi)}+\mu^{H} \nabla_{\bar{z}} h(\xi)\right] \\
<-\left(\frac{\rho_{1}}{\alpha_{1}(z, u)}+\frac{\rho_{2}}{\alpha_{2}(z, u)}\right) \theta^{2}(z, u) .
\end{gathered}
$$

From the assumption $\rho_{1} / \alpha_{1}(z, u)+\rho_{2} / \alpha_{2}(z, u) \geq 0$, the above inequality yields

$$
\begin{gathered}
F\left[z, u ; \sum_{i=1}^{k} \lambda_{i}\left\{\overline{\nabla_{z} f\left(\xi, \eta_{i}\right)}+\nabla_{\bar{z}} f\left(\xi, \eta_{i}\right)+A w\right\}\right. \\
\left.+\mu^{T} \overline{\nabla_{z} h(\xi)}+\mu^{H} \nabla_{\bar{z}} h(\xi)\right]<0,
\end{gathered}
$$

which contradicts (80), hence the theorem.
Theorem 22 (weak duality). Let $\zeta=(z, \bar{z})$ and $(k, \tilde{\lambda}, \tilde{\eta}, \xi, \mu, w)$ be feasible solutions to $(P)$ and (D2), respectively. Further, if $\operatorname{Re}\left[\sum_{i=1}^{k} \lambda_{i}\left[f\left(\zeta, \eta_{i}\right)+z^{H} A w\right]\right]$ is $\left(F, \alpha_{1}, \rho_{1}, \theta\right)$-quasiconvex with respect to $R_{+}$on $Q, h(\zeta)$ is $\left(F, \alpha_{2}, \rho_{2}, \theta\right)$-pseudoconvex on $Q$ with respect to the polyhedral cone $S \subset C^{p}$, and $\rho_{1} / \alpha_{1}(z, u)+\rho_{2} / \alpha_{2}(z, u) \geq 0$, then

$$
\begin{aligned}
& \sup _{\tilde{\eta} \in Y} \operatorname{Re}\left[f(\zeta, \tilde{\eta})+\left(z^{H} A z\right)^{1 / 2}\right] \\
& \quad \geq \sup _{\tilde{\eta} \in Y} \operatorname{Re}\left[f(\xi, \tilde{\eta})+\left(u^{H} A u\right)^{1 / 2}\right] .
\end{aligned}
$$

Proof. The proof follows the same lines as in Theorem 21.

Theorem 23 (strong duality). Let $\zeta_{0}=\left(z_{0}, \bar{z}_{0}\right)$ be an optimal solution to the problem $(P)$ at which a constraint qualification is a satisfied. Then there exist $(k, \tilde{\lambda}, \tilde{\eta}) \in K\left(\zeta_{0}\right)$ and $\left(\zeta_{0}, \mu, w\right) \in$ $X(k, \tilde{\lambda}, \widetilde{\eta})$ such that $\left(k, \tilde{\lambda}, \widetilde{\eta}, \zeta_{0}, \mu, w\right)$ is a feasible solution to the dual problem (D2). Further, if the hypotheses of Theorem 21 or Theorem 22 are satisfied, then $\left(k, \widetilde{\lambda}, \widetilde{\eta}, \zeta_{0}, \mu, w\right)$ is optimal to (D2), and the two problems $(P)$ and (D2) have the same optimal values.

Proof. The proof follows along the lines of Theorem 8 (Lai and Liu [13]).

Theorem 24 (strict converse duality). Let $\hat{\zeta}$ and $(\widehat{k}, \widehat{\tilde{\lambda}}, \widehat{\tilde{\eta}}, \widehat{\xi}, \widehat{\mu}, \widehat{w})$ be optimal solutions to $(P)$ and $(D 2)$, respectively, and the conditions of Theorem 23 are satisfied. Further, assume that the following conditions are satisfied:

(i) $\operatorname{Re} \sum_{i=1}^{\widehat{k}} \hat{\lambda}_{i}\left[f\left(\widehat{\zeta}, \widehat{\eta}_{i}\right)+z^{H} A \widehat{w}\right]$ is strict $\left(F, \alpha_{1}, \rho_{1}, \theta\right)-$ pseudoconvex with respect to $R_{+}$on $Q$ and $h(\widehat{\zeta})$ is $\left(F, \alpha_{2}, \rho_{2}, \theta\right)$-quasiconvex on $Q$ with respect to the polyhedral cone $S \subset C^{p}$;

(ii) $\rho_{1} / \alpha_{1}(\widehat{z}, \widehat{u})+\rho_{2} / \alpha_{2}(\widehat{z}, \widehat{u}) \geq 0$.

Then $\widehat{\zeta}=\widehat{\xi}$; that is, $\widehat{\xi}$ is an optimal solution to $(D 2)$.

Proof. On the contrary, we assume that $(\widehat{z}, \overline{\widehat{z}})=\widehat{\zeta} \neq \widehat{\xi}=(\widehat{u}, \overline{\widehat{u}})$. On applying Theorem 23, we know that

$$
\begin{aligned}
& \sup _{\tilde{\tilde{\eta}} \in Y} \operatorname{Re}\left[f(\widehat{\zeta}, \widehat{\tilde{\eta}})+\left(\widehat{z}^{H} A \widehat{z}\right)^{1 / 2}\right] \\
& =\sup _{\widehat{\tilde{\eta}} \in Y} \operatorname{Re}\left[f(\widehat{\xi}, \widehat{\tilde{\eta}})+\left(\widehat{u}^{H} A \widehat{u}\right)^{1 / 2}\right] .
\end{aligned}
$$

From the feasibility of $\widehat{\zeta} \in Q$ to $(\mathrm{P}), \widehat{\mu} \in S^{*}$, inequality (81) yields

$$
\operatorname{Re}\langle h(\widehat{\zeta}), \widehat{\mu}\rangle \leq 0 \leq \operatorname{Re}\langle h(\widehat{\xi}), \widehat{\mu}\rangle .
$$

Since $h(\widehat{\zeta})$ is $\left(F, \alpha_{2}, \rho_{2}, \theta\right)$-quasiconvex on $Q$ with respect to the polyhedral cone $S$ in $C^{p}$, the above inequality yields

$$
\begin{aligned}
& F\left[\widehat{z}, \widehat{u} ; \alpha_{2}(\widehat{z}, \widehat{u})\left(\widehat{\mu}^{T} \overline{\nabla_{z} h(\widehat{\xi})}+\widehat{\mu}^{H} \nabla_{\bar{z}} h(\widehat{\xi})\right)\right] \\
& \quad \leq-\rho_{2} \theta^{2}(\widehat{z}, \widehat{u}),
\end{aligned}
$$


which by sublinearity of $F$ implies

$$
\begin{aligned}
& F\left[\widehat{z}, \widehat{u} ;\left(\widehat{\mu}^{T} \overline{\nabla_{z} h(\widehat{\xi})}+\widehat{\mu}^{H} \nabla_{\bar{z}} h(\widehat{\xi})\right)\right] \\
& \quad \leq-\frac{\rho_{2}}{\alpha_{2}(\widehat{z}, \widehat{u})} \theta^{2}(\widehat{z}, \widehat{u}) .
\end{aligned}
$$

By (80) and the sublinearity of $F$, we have

$$
\begin{aligned}
& F\left[\widehat{z}, \widehat{u} ; \sum_{i=1}^{\widehat{k}} \hat{\lambda}_{i}\left[\overline{\nabla_{z} f\left(\widehat{\xi}, \widehat{\eta}_{i}\right)}+\nabla_{\bar{z}} f\left(\widehat{\xi}, \widehat{\eta}_{i}\right)+A \widehat{w}\right]\right] \\
& +F\left[\widehat{z}, \widehat{u} ;\left(\widehat{\mu}^{T} \overline{\nabla_{z} h(\widehat{\xi})}+\widehat{\mu}^{H} \nabla_{\bar{z}} h(\widehat{\xi})\right)\right] \\
& \geq F\left[\widehat{z}, \widehat{u} ; \sum_{i=1}^{\widehat{k}} \hat{\lambda}_{i}\left[\overline{\nabla_{z} f\left(\widehat{\xi}, \widehat{\eta}_{i}\right)}+\nabla_{\bar{z}} f\left(\widehat{\xi}, \widehat{\eta}_{i}\right)+A \widehat{w}\right]\right. \\
& \left.\quad+\widehat{\mu}^{T} \overline{\nabla_{z} h(\widehat{\xi})}+\widehat{\mu}^{H} \nabla_{\bar{z}} h(\widehat{\xi})\right] \\
& =0 .
\end{aligned}
$$

The above inequality, together with (102) and $\rho_{1} / \alpha_{1}(\widehat{z}, \widehat{u})+$ $\rho_{2} / \alpha_{2}(\widehat{z}, \widehat{u}) \geq 0$, gives

$$
\begin{aligned}
& F\left[\widehat{z}, \widehat{u} ; \sum_{i=1}^{\widehat{k}} \widehat{\lambda}_{i}\left[\overline{\nabla_{z} f\left(\widehat{\xi}, \widehat{\eta}_{i}\right)}+\nabla_{\bar{z}} f\left(\widehat{\xi}, \widehat{\eta}_{i}\right)+A \widehat{w}\right]\right] \\
& \quad \geq-F\left[\widehat{z}, \widehat{u} ;\left(\widehat{\mu}^{T} \overline{\nabla_{z} h(\widehat{\xi})}+\widehat{\mu}^{H} \nabla_{\bar{z}} h(\widehat{\xi})\right)\right] \\
& \quad \geq \frac{\rho_{2}}{\alpha_{2}(\widehat{z}, \widehat{u})} \theta^{2}(\widehat{z}, \widehat{u}) \\
& \quad \geq-\frac{\rho_{1}}{\alpha_{1}(\widehat{z}, \widehat{u})} \theta^{2}(\widehat{z}, \widehat{u}) .
\end{aligned}
$$

That is,

$$
\begin{aligned}
& F\left[\widehat{z}, \widehat{u} ; \sum_{i=1}^{\widehat{k}} \widehat{\lambda}_{i}\left[\overline{\nabla_{z} f\left(\widehat{\xi}, \widehat{\eta}_{i}\right)}+\nabla_{\bar{z}} f\left(\widehat{\xi}, \widehat{\eta}_{i}\right)+A \widehat{w}\right]\right] \\
& \quad \geq-\frac{\rho_{1}}{\alpha_{1}(\widehat{z}, \widehat{u})} \theta^{2}(\widehat{z}, \widehat{u}),
\end{aligned}
$$

which by sublinearity of $F$ implies

$$
\begin{aligned}
& F\left[\widehat{z}, \widehat{u} ; \alpha_{1}(\widehat{z}, \widehat{u}) \sum_{i=1}^{\hat{k}} \widehat{\lambda}_{i}\left[\overline{\nabla_{z} f\left(\widehat{\xi}, \widehat{\eta}_{i}\right)}+\nabla_{\bar{z}} f\left(\widehat{\xi}, \widehat{\eta}_{i}\right)+A \widehat{w}\right]\right] \\
& \quad \geq-\rho_{1} \theta^{2}(\widehat{z}, \widehat{u}) .
\end{aligned}
$$

Since $\operatorname{Re} \sum_{i=1}^{\widehat{k}} \hat{\lambda}_{i}\left[f\left(\widehat{\zeta}, \widehat{\eta}_{i}\right)+z^{H} A \widehat{w}\right]$ is strict $\left(F, \alpha_{1}, \rho_{1}, \theta\right)$ pseudoconvex with respect to $R_{+}$on $Q$, the above inequality implies that

$$
\operatorname{Re}\left[\sum_{i=1}^{\widehat{k}} \widehat{\lambda}_{i}\left[f\left(\widehat{\zeta}, \widehat{\eta}_{i}\right)+\widehat{z}^{H} A \widehat{w}\right]\right]
$$

$$
>\operatorname{Re}\left[\sum_{i=1}^{\widehat{k}} \widehat{\lambda}_{i}\left[f\left(\widehat{\xi}, \widehat{\eta}_{i}\right)+\widehat{u}^{H} A \widehat{w}\right]\right] .
$$

From (82), (83), and the generalized Schwarz inequality, we get

$$
\operatorname{Re}\left(\widehat{z}^{H} A \widehat{w}\right) \leq\left(\widehat{z}^{H} A \widehat{z}\right)^{1 / 2}, \quad \operatorname{Re}\left(\widehat{u}^{H} A \widehat{w}\right)^{1 / 2}=\left(\widehat{u}^{H} A \widehat{u}\right)^{1 / 2}
$$

which on substituting in (107), we obtain

$$
\begin{gathered}
\operatorname{Re}\left[\sum_{i=1}^{\widehat{k}} \hat{\lambda}_{i}\left[f\left(\widehat{\zeta}, \widehat{\eta}_{i}\right)+\left(\hat{z}^{H} A \widehat{z}\right)^{1 / 2}\right]\right] \\
>\operatorname{Re}\left[\sum_{i=1}^{\hat{k}} \hat{\lambda}_{i}\left[f\left(\widehat{\xi}, \widehat{\eta}_{i}\right)+\left(\widehat{u}^{H} A \widehat{u}\right)^{1 / 2}\right]\right] .
\end{gathered}
$$

Consequently, there exist certain $i_{0}$ which satisfy

$$
\begin{aligned}
& \operatorname{Re}\left[f\left(\widehat{\zeta}, \widehat{\eta}_{i_{0}}\right)+\left(\widehat{z}^{H} A \widehat{z}\right)^{1 / 2}\right] \\
& >\operatorname{Re}\left[f\left(\widehat{\xi}, \widehat{\eta}_{i_{0}}\right)+\left(\widehat{u}^{H} A \widehat{u}\right)^{1 / 2}\right] .
\end{aligned}
$$

Hence,

$$
\begin{aligned}
& \sup _{\widehat{\tilde{\eta}} \in Y} \operatorname{Re}\left[f(\widehat{\zeta}, \widehat{\widetilde{\eta}})+\left(\widehat{z}^{H} A \widehat{z}\right)^{1 / 2}\right] \\
& \geq \operatorname{Re}\left[f\left(\widehat{\zeta}, \widehat{\eta}_{i_{0}}\right)+\left(\widehat{z}^{H} A \widehat{z}\right)^{1 / 2}\right] \\
& >\operatorname{Re}\left[f\left(\widehat{\xi}, \widehat{\eta}_{i_{0}}\right)+\left(\widehat{u}^{H} A \widehat{u}\right)^{1 / 2}\right] \\
& =\sup _{\widetilde{\tilde{\eta}} \in Y} \operatorname{Re}\left[f(\widehat{\xi}, \widehat{\tilde{\eta}})+\left(\widehat{u}^{H} A \widehat{u}\right)^{1 / 2}\right],
\end{aligned}
$$

which contradicts (99), hence the theorem.

\section{Conclusion}

In this paper, we introduced generalized $(F, \alpha, \rho, \theta)$-convex functions and established sufficient optimality conditions for a class of nondifferentiable minimax programming problems in complex space. These optimality conditions are then used to construct two types of dual model and finally we derived weak, strong, and strict converse duality theorems 
to show that there is no duality gap between the two dual problems with respect to the primal problem under some generalized convexities of complex functions in the complex programming problem. As a future task, the authors would like to extend these results to second and higher order cases and establish the relations between primal and its second and higher order dual problems.

\section{Acknowledgments}

The authors are thankful to the anonymous referees for their valuable comments which have improved the presentation of the paper. The research of the first author is financially supported by the University Grant Commission, New Delhi, India, through Grant No. F. no. 41-801/2012(SR).

\section{References}

[1] N. Levinson, "Linear programming in complex space," Journal of Mathematical Analysis and Applications, vol. 14, no. 1, pp. 4462, 1966.

[2] J. C. Chen and H. C. Lai, "Optimality conditions for minimax programming of analytic functions," Taiwanese Journal of Mathematics, vol. 8, no. 4, pp. 673-686, 2004.

[3] N. Datta and D. Bhatia, "Duality for a class of nondifferentiable mathematical programming problems in complex space," Journal of Mathematical Analysis and Applications, vol. 101, no. 1, pp. $1-11,1984$.

[4] O. P. Jain and P. C. Saxena, "A duality theorem for a special class of programming problems in complex space," Journal of Optimization Theory and Applications, vol. 16, no. 3-4, pp. 207220, 1975.

[5] I. M. Stancu-Minasian, Fractional Programming: Theory, Methods and Applications, vol. 409, Kluwer Academic, Dordrecht, Germany, 1997.

[6] I. Ahmad and Z. Husain, "Optimality conditions and duality in nondifferentiable minimax fractional programming with generalized convexity," Journal of Optimization Theory and Applications, vol. 129, no. 2, pp. 255-275, 2006.

[7] A. Jayswal, I. Ahmad, and D. Kumar, "Nondifferentiable minimax fractional programming problem with nonsmooth generalized convex functions," Communications on Applied Nonlinear Analysis, vol. 18, no. 4, pp. 57-75, 2011.

[8] A. Jayswal and D. Kumar, "On nondifferentiable minimax fractional programming involving generalized (C, $\alpha, \rho, d)$ convexity," Communications on Applied Nonlinear Analysis, vol. 18, no. 1, pp. 61-77, 2011.

[9] H.-C. Lai, J.-C. Lee, and S.-C. Ho, "Parametric duality on minimax programming involving generalized convexity in complex space," Journal of Mathematical Analysis and Applications, vol. 323, no. 2, pp. 1104-1115, 2006.

[10] K. Swarup and I. C. Sharma, "Programming with linear fractional functionals in complex space," Cahiers Center d'Etudes Recherche Operationelle, vol. 12, pp. 103-109, 1970.

[11] A. Charnes and W. W. Cooper, "Programming with linear fractional functionals," Naval Research Logistics Quarterly, vol. 9, no. 3-4, pp. 181-186, 1962.

[12] H.-C. Lai and T.-Y. Huang, "Optimality conditions for nondifferentiable minimax fractional programming with complex variables," Journal of Mathematical Analysis and Applications, vol. 359, no. 1, pp. 229-239, 2009.
[13] H.-C. Lai and J.-C. Liu, "Duality for nondifferentiable minimax programming in complex spaces," Nonlinear Analysis: Theory, Methods and Applications, vol. 71, no. 12, pp. e224-e233, 2009.

[14] I. Ahmad, Z. Husain, and S. Sharma, "Second-order duality in nondifferentiable minmax programming involving type-I functions," Journal of Computational and Applied Mathematics, vol. 215, no. 1, pp. 91-102, 2008.

[15] I. Ahmad, Z. Husain, and S. Sharma, "Higher-order duality in nondifferentiable minimax programming with generalized type I functions," Journal of Optimization Theory and Applications, vol. 141, no. 1, pp. 1-12, 2009.

[16] B. Mond and B. D. Craven, "A class of nondifferentiable complex programming problems," Journal of Mathematics and Statistics, vol. 6, pp. 581-591, 1975.

[17] S. K. Mishra and N. G. Rueda, "Symmetric duality for mathematical programming in complex spaces with F-convexity," Journal of Mathematical Analysis and Applications, vol. 284, no. 1, pp. 250-265, 2003.

[18] H. C. Lai and J. C. Liu, "Minimax programming in complex spaces-necessary and sufficient optimality conditions," in Proceedings of the International Symposium on Nonlinear Analysis and Convex Analysis, 2007, Research Institute for Mathematical Sciences, Kyoto University, Japan Rims Kokuroku, 2008. 


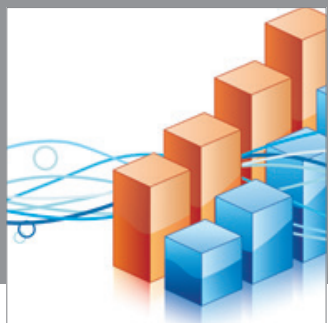

Advances in

Operations Research

mansans

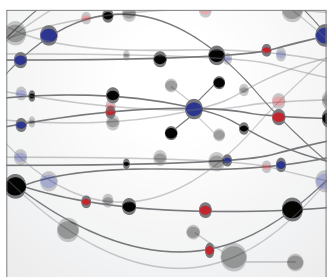

The Scientific World Journal
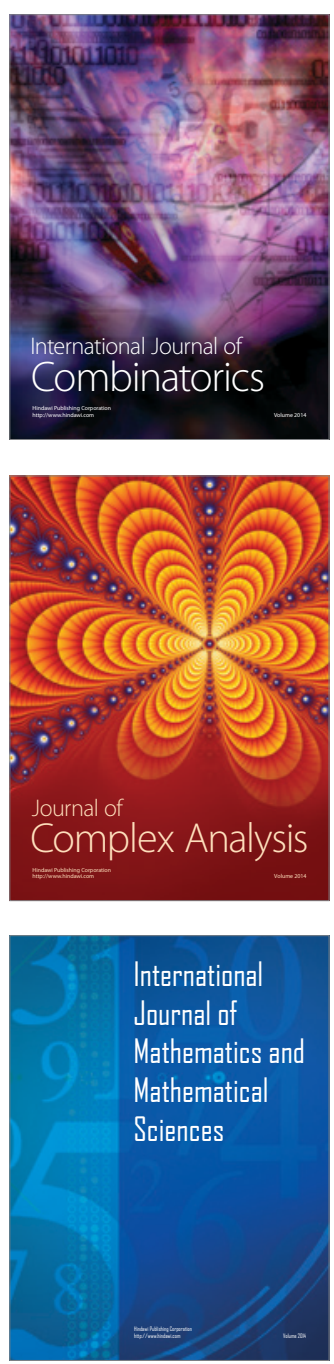
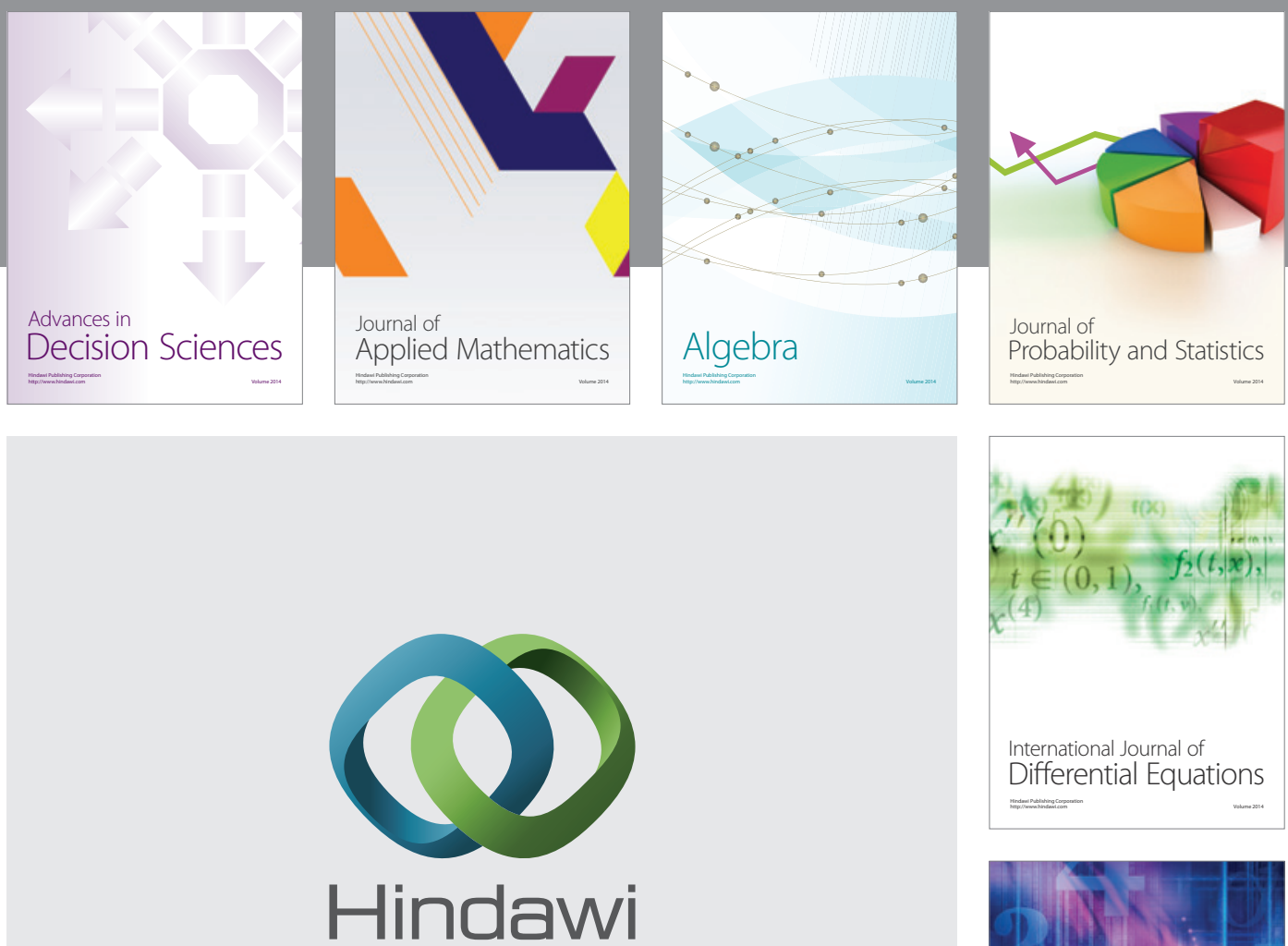

Submit your manuscripts at http://www.hindawi.com
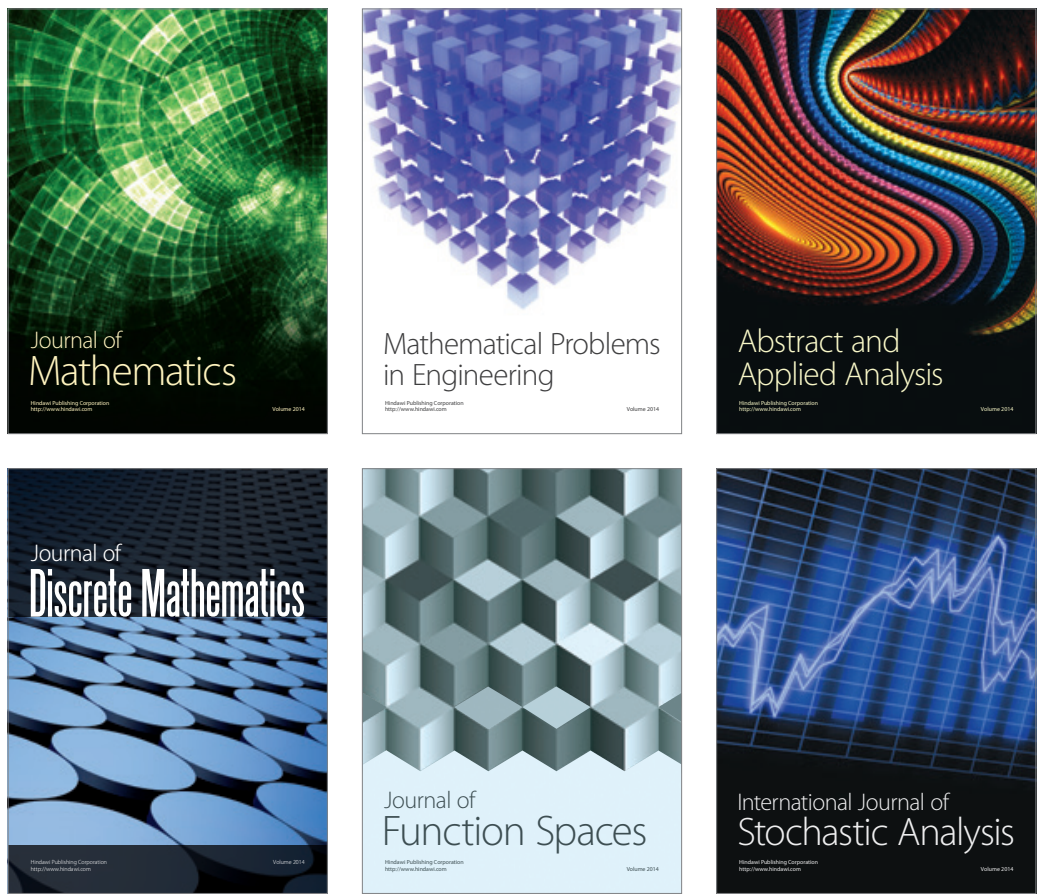

Journal of

Function Spaces

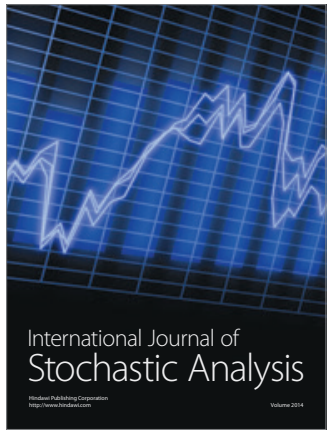

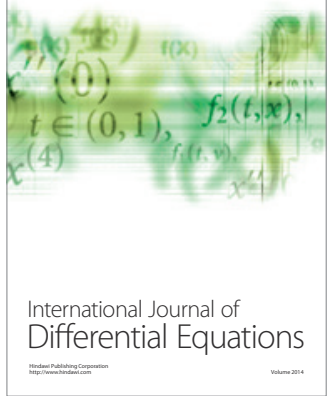
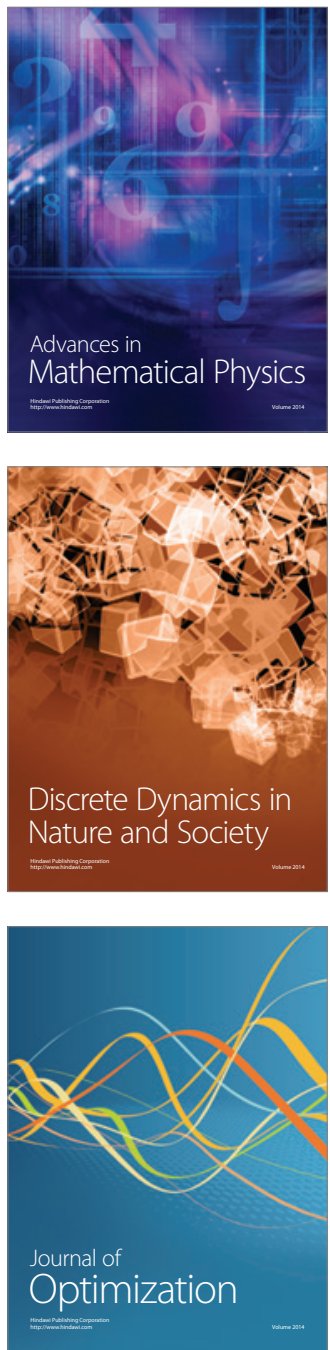